\title{
Genus 3 normal coverings of the Riemann sphere branched over 4 points
}

\section{Yolanda Fuertes and Manfred Streit}

\begin{abstract}
In this paper we study the 5 families of genus 3 compact Riemann surfaces which are normal coverings of the Riemann sphere branched over 4 points from very different aspects: their moduli spaces, the uniform Belyi functions that factorize through the quotient by the automorphism groups and the Weierstrass points of the non hyperelliptic families.
\end{abstract}

\section{Introduction}

The genus 3 complex algebraic curves or compact Riemann surfaces which are normal coverings of the Riemann sphere branched over four points belong to 3 different non-hyperelliptic families and 2 different hyperelliptic families already known since the work of Broughton [2] and Kuribayashi and Komiya [10]. The former describes the finite groups that occur as automorphism groups for genus 2 and 3 compact Riemann surfaces in the setting of Fuchsian groups, whereas Kuribayashi and Komiya obtain the algebraic equations for the families of genus 3 curves and the automorphism groups they admit. Here we make explicit not only the canonical models for the non-hyperelliptic families, but also for every family the morphism or meromorphic function to the Riemann sphere corresponding to taking the quotient by its automorphism group. This is done mainly combining representation and character theory with classical results about compact Riemann surfaces, as developed in the second author's paper [16]. At this point, after a straightforward analysis of the cross ratios of the 4 branched points, we describe their isomorphisms classes or moduli spaces. The articles [6], [4] or [12] are among a wide list of papers amid by the study of the moduli spaces of families of curves having certain automorphism groups.

2000 Mathematics Subject Classification: 14H15, 14H45, $14 \mathrm{H} 55$.

Keywords: Moduli of algebraic curves with automorphisms, Weierstrass points, uniform Belyi functions. 
We obtain as well the curves inside each family that admit a uniform Belyi function (that is, a meromorphic function having at most three critical values and with the property that every point on the fiber of each critical value has the same ramification index or, equivalently, these compact Riemann surfaces have the property of satisfying an algebraic equation defined over a number field and they have a Belyi function that provides a uniformization in the classical sense via its monodromy representation) which factorize through the quotient by the automorphism group (see [15] for the description of the genus 1 curves with a uniform Belyi function). Finally, we find the Weierstrass points for every curve belonging to the non-hyperelliptic families, giving explicitly the Wronskians for suitable basis of the spaces of holomorphic differentials. The Weierstrass points are characterized by the property of being zeros of order $\geq g$ of a holomorphic differential. For every curve of genus $g \geq 2$, the number of Weierstrass points is in between $2 g+2$ and $g(g-1)(g+1)$. Hyperelliptic curves are the ones having exactly $2 g+2$ Weierstrass points. However, in general it is not an easy issue to find the Weierstrass points of a curve; for instance, it is still an open problem to find all the Weierstrass points of the Fermat curves $x^{n}+y^{n}=1$ for $n>6$ (see [13] for a survey on this).

Acknowledgment. The first author is indebted to D. Singerman for oral communication of Lemma 3 and for supplying very useful references as well as to G. A. Jones for its valuable suggestions about some group theoretical aspects of the paper. We are grateful to G. González-Díez for asking us about the moduli of these families and J. Wolfart for interesting discussions related with the subject.

\section{Preliminaries results}

According to Broughton's list [2] in terms of Fuchsian groups or Kuribayashi and Komiya [10] in terms of algebraic curves, there exist the following 1complex parameter families of genus 3 compact Riemann surfaces admitting an automorphism group $G$, for which the morphisms which project to the quotient surfaces under the action of the automorphism group $G$, are meromorphic functions branched over 4 points, the branching orders specified below:

$\begin{array}{rcccc} & \text { Algebraic equation } & \mathrm{G} & \text { Branch orders } \\ \text { (I) } & \mathcal{F}_{1}: & x^{4}+y^{4}+t\left(x^{2} y^{2}+x^{2}+y^{2}\right)=-1 & \mathrm{~S}_{4} & (2,2,2,3) \\ (\mathrm{II}) & \mathcal{F}_{2}: & y^{4}=x^{4}-t x^{2}+1 & \mathrm{C}_{2} \ltimes\left(\mathrm{C}_{2} \times \mathrm{C}_{4}\right) & (2,2,2,4) \\ (\mathrm{III}) & \mathcal{F}_{3}: & y^{2}=x^{8}-t x^{4}+1 & \mathrm{D}_{4} \times \mathrm{C}_{2} & (2,2,2,4) \\ (\mathrm{IV}) & \mathcal{F}_{4}: & y^{2}=x^{7}-t x^{4}+x & \mathrm{D}_{6} & (2,2,2,6) \\ (\mathrm{V}) & \mathcal{F}_{5}: & y^{3}=\left(x^{2}-1\right)\left(x^{2}-t\right) & \mathrm{C}_{6} & (2,3,3,6) .\end{array}$


Before starting with the analysis and description of each case and for the sake of completeness, let us state some notation and elementary facts about normal coverings in the framework of Fuchsian groups and rewrite, for our particular situation, Lemma 5.2 in [6].

Due to the Uniformization Theorem, an arbitrary Riemann surface of genus $g>1 S$ can be viewed as a quotient space $S \cong \mathbb{H} / K$, where $\mathbb{H}$ is the upper half-plane and $K$ is the uniformizing group, a discrete subgroup of $P S L_{2}(\mathbb{R})$, the group of conformal automorphisms of $\mathbb{H}$, which is torsion free and acts freely on $\mathbb{H}$, isomorphic to the fundamental group of the surface and very often termed a (Fuchsian) surface group. From elementary facts of covering space theory, every conformal automorphism $\alpha: S \rightarrow S$ lifts to a conformal automorphism of $\mathbb{H}$, $\widetilde{\alpha}$, such that $\widetilde{\alpha} K \widetilde{\alpha}^{-1}=K$, i.e: $\widetilde{\alpha}$ belongs to the normalizer of $K$ in $P S L_{2}(\mathbb{R})$, let us denote it from now on by $N(K)$, and vice versa. With the analogous notation, the existence of a compact Riemann surface of genus $g>1, S \cong \mathbb{H} / K$, admitting an automorphism group isomorphic to $G$ with quotient surface $S / G \cong S_{1} \cong \mathbb{H} / \Gamma$ (that is, $\quad \Gamma=<K, \widetilde{G}>$ ) is, obviously, equivalent to the existence of a surface kernel epimorphism $\varphi: \Gamma \rightarrow G$ (that is, $\operatorname{Kernel}(\varphi)$ is a surface group) and $K=\operatorname{Kernel}(\varphi)$. We will say that two surface kernel epimorphisms are equivalent if they differ by post-composition with an automorphism of $G$.

The signature $\left[\gamma ; m_{1}, m_{2}, \ldots, m_{r}\right]$ of the Fuchsian group $\Gamma$ encodes its presentation as an abstract group as well as the geometric nature of the action of $G: \gamma$ is the genus of the quotient surface $S_{1}$ and the $\left\{m_{i}\right\}$ are the branching orders of the covering $S \rightarrow S_{1}$ (for details see [9]).

From now on we will refer to $K \unlhd \Gamma$ as the normal inclusion of Fuchsian groups that realizes the covering $S \longrightarrow S / G$ and to $G$ as the covering group. By other hand, we will use the standard abbreviated notation $\left[m_{1}, m_{2}, \ldots, m_{r}\right]$ when $\gamma=0$.

Lemma 1. Let $S \cong \mathbb{H} / K$ and $S^{\prime} \cong \mathbb{H} / K^{\prime}$ be compact Riemann surfaces of genus $g>1$, normal coverings of the Riemann sphere $\mathbb{C P}^{1}$ branched over $\left\{a_{1}, a_{2}, a_{3}, a_{4}\right\}$ and $\left\{a_{1}^{\prime}, a_{2}^{\prime}, a_{3}^{\prime}, a_{4}^{\prime}\right\}$, respectively, with covering group $G ; K$ and $K^{\prime}$ surface groups, $K \unlhd \Gamma$ and $K^{\prime} \unlhd \Gamma^{\prime}$ the normal inclusions that realize the coverings, and let us denote by $\varphi$ and $\varphi^{\prime}$ the surface kernel epimorphisms, respectively. Then, a conformal automorphism $M: \mathbb{C P}^{1} \rightarrow \mathbb{C P}^{1}$ with $M\left(a_{i}\right)=a_{i}^{\prime}$ lifts to a conformal automorphism between $S$ and $S^{\prime}$ if and only if there exists an automorphism of $G, \tau$, such that $\varphi^{\prime} \circ \eta=\tau \circ \varphi$, where $\eta$ is the isomorphism between the groups $\Gamma$ and $\Gamma^{\prime}$ induced by $M$.

Proof. This lemma is a corollary of Lemma 5.2 in [6] which is the general result. 
Remark 2. For the well known equivalence between compact Riemann surfaces or algebraic curves and their algebraic function fields, morphisms $f: S \rightarrow S^{\prime}$ correspond to function field extensions of degree equal to the degree of the morphisms, that is, $f^{*}\left(\mathbb{C}\left(S^{\prime}\right)\right) \hookrightarrow \mathbb{C}(S)$ and $\left[\mathbb{C}(S): f^{*}\left(\mathbb{C}\left(S^{\prime}\right)\right]=\right.$ $\operatorname{deg}(f)$. In particular, normal coverings with covering group $G$ correspond to Galois function field extensions and, of course, the function field of the quotient surface is the fixed field under the action of the Galois group of the extension; namely, $\mathbb{C}\left(S^{\prime}\right) \cong(\mathbb{C}(S))^{G}$. We refer to [5] for basic and general background about algebraic curves or compact Riemann surfaces and their morphisms.

\section{Case (I). Moduli of the family $\mathcal{F}_{1}$}

The 1-complex dimensional family of genus 3 compact Riemann surfaces admitting an automorphism group isomorphic to $S_{4}$ with branching data $(2,2,2,3), \mathcal{F}_{1}$, was first studied by Wiman in [18] and later on, among others, by Rodríguez and González-Aguilera in [12].

For every $t \in \mathbb{C}-\{ \pm 2,-1\}$, the corresponding smooth projective curve is given by (see e.g. [12])

$$
S_{t} \equiv\left\{x^{4}+y^{4}+z^{4}+t\left(x^{2} y^{2}+x^{2} z^{2}+y^{2} z^{2}\right)=0\right\} \subseteq \mathbb{C P}^{2}
$$

and, in fact, this is a canonical model for each associated genus 3 compact Riemann surface in $\mathcal{F}_{1}$. In the following brief account we try to give as explicit as possible the connection between the surface kernel construction and the canonical model though most of the information are already available in the cited papers, but we feel that this might be helpful for a better understanding of the following.

In order to calculate the number of different epimorphisms $\varphi$ from a Fuchsian quadrangle group of signature $[2,2,2,3], \Gamma=\langle x, y, z| x^{2}=y^{2}=$ $\left.z^{2}=(x y z)^{-3}=1\right\rangle$, to the finite group $S_{4}$, the character table of $S_{4}$ is helpful

$\begin{array}{cccccc}C & 1 A & 2 A & 2 B & 3 A & 4 A \\ |C| & 1 & 6 & 3 & 8 & 6 \\ & () & (12) & (12)(34) & (123) & (1234) \\ \psi_{1} & 1 & 1 & 1 & 1 & 1 \\ \psi_{2} & 1 & -1 & 1 & 1 & -1 \\ \psi_{3} & 2 & 0 & 2 & -1 & 0 \\ \psi_{4} & 3 & 1 & -1 & 0 & -1 \\ \psi_{5} & 3 & -1 & -1 & 0 & 1\end{array}$

where we use the standard ATLAS notation. For parity reasons two of the order two elements $x, y$ and $z$ should be mapped to elements of the 
classes $2 A$ where the remaining element should be mapped to an element of the class $2 B$. Notice also that a subgroup of $S_{4}$ containing both a transposition and a three cycle is the whole group. Thus the number of different epimorphisms equals the number of solutions of the equations $\bar{x}_{1}^{2}=\bar{x}_{2}^{2}=\bar{x}_{3}^{2}=\bar{x}_{4}^{3}=\bar{x}_{1} \bar{x}_{2} \bar{x}_{3} \bar{x}_{4}=1, \bar{x}_{i} \in S_{4}$. This number $n_{\varphi}$ can be easily calculated by using the well known formula, c.f. [8]

$$
n_{\varphi}:=3 \frac{|2 A||| 2 A|| 2 B|| 3 A \mid}{\left|S_{4}\right|} \sum_{i=1}^{5} \frac{\psi_{i}\left(\bar{x}_{1}\right) \psi_{i}\left(\bar{x}_{2}\right) \psi_{i}\left(\bar{x}_{3}\right) \psi_{i}\left(\bar{x}_{4}\right)}{\psi_{i}(1)^{2}}=216 .
$$

Of course to obtain the number of different surface kernels we have to bring into account that two chosen image quadruples lead to the same surface kernel if and only if they differ by an automorphism of the group $S_{4}$. Being a complete group, i.e. $\operatorname{Aut}\left(S_{4}\right)=S_{4}$, we obtain the total number of different kernels inside the $[2,2,2,3]$ quadrangle group to be $n_{\varphi} / 24=9$. It is easy to verify that the epimorphisms which lead to different surface kernels can be chosen in the following way:

Lemma 3. For every fuchsian group $\Gamma$ with signature $[2,2,2,3]$, there exist the following 9 non-equivalent surface epimorphisms $\varphi_{l}^{j}: \Gamma \rightarrow S_{4}(l, j=$ 1, 2 and 3$)$ :

$$
\begin{array}{lll}
\varphi_{1}^{1}(x)=(12)(34) & \varphi_{1}^{2}(x)=(14)(23) & \varphi_{1}^{3}(x)=(13)(24) \\
\varphi_{1}^{1}(y)=(12) & \varphi_{1}^{2}(y)=(12) & \varphi_{1}^{3}(y)=(12) \\
\varphi_{1}^{1}(z)=(23) & \varphi_{1}^{2}(z)=(23) & \varphi_{1}^{3}(z)=(23) \\
& & \\
\varphi_{2}^{1}(x)=(12) & \varphi_{2}^{2}(x)=(12) & \varphi_{2}^{3}(x)=(12) \\
\varphi_{2}^{1}(y)=(12)(34) & \varphi_{2}^{2}(y)=(14)(23) & \varphi_{2}^{3}(y)=(13)(24) \\
\varphi_{2}^{1}(z)=(23) & \varphi_{2}^{2}(z)=(23) & \varphi_{2}^{3}(z)=(23) \\
\varphi_{3}^{1}(x)=(12) & \varphi_{3}^{2}(x)=(12) & \varphi_{3}^{3}(x)=(12) \\
\varphi_{3}^{1}(y)=(23) & \varphi_{3}^{2}(y)=(23) & \varphi_{3}^{3}(y)=(23) \\
\varphi_{3}^{1}(z)=(12)(34) & \varphi_{3}^{2}(z)=(14)(23) & \varphi_{3}^{3}(z)=(13)(24) .
\end{array}
$$

Proof. None of the triples are simultaneously conjugate.

Generically these kernels belong to biholomorphically inequivalent or non isomorphic Riemann surfaces. However it might occur that some of these kernels are conjugate inside $P S L_{2}(\mathbb{R})$, that is, in some bigger Fuchsian group $\Delta$ containing the group $[2,2,2,3]$. It is an easy observation that $\Delta$ has to be a triangle group. 
Lemma 4. a) The possible inclusions of a Fuchsian group of signature $[2,2,2,3]$ inside a triangle group $\Delta_{i}$ are the following

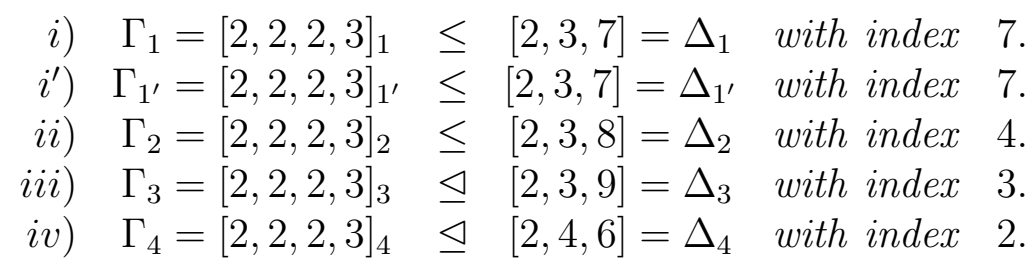

b) In case iii) the nine different kernels belong to only three biholomorphically inequivalent Riemann surfaces, i.e. the orbit of a kernel inside [2,3,9] under conjugation has length 3. In case iv) we find 5 different Riemann surfaces. In case $i), i^{\prime}$ ) and $\left.i i\right)$ there are 9 biholomorphically inequivalent Riemann surfaces.

Proof. a) The proof that those triangle groups are the only possible funchsian groups which contain a quadrangle group with signature $[2,2,2,3]$ is done in [3] using a simple area argument. According to Theorem 1 in [14], the existence of the above inclusions of Fuchsian groups comes from the existence of the following permutation representations $\theta_{i}$ of the action of the generators of the corresponding triangle group, $\Delta_{i}$, on the set of left coset representatives of $\Delta_{i} / \Gamma_{i}$, for $i=1,2,3$ and 4; namely:

$$
\begin{aligned}
& \theta_{1}:[2,3,7] \rightarrow S_{7} \\
& x_{1} \rightarrow(37)(56)(1)(2)(4) \\
& y_{1} \rightarrow(132)(475) \\
& z_{1} \rightarrow(1234567) \\
& \theta_{1^{\prime}}:[2,3,7] \rightarrow S_{7} \\
& x_{1}^{\prime} \rightarrow(1)(2)(6)(37)(45) \\
& y_{1}^{\prime} \rightarrow(132)(476) \\
& z_{1}^{\prime} \rightarrow(1234567) \\
& \theta_{2}:[2,3,8] \rightarrow S_{4} \\
& x_{2} \rightarrow(1)(2)(34) \\
& \theta_{3}:[2,3,9] \rightarrow S_{3} \\
& x_{3} \rightarrow(1)(2)(3) \\
& \theta_{4}:[2,4,6] \rightarrow S_{2} \\
& y_{2} \rightarrow(4)(132) \\
& y_{3} \rightarrow(123) \\
& x_{4} \rightarrow(1)(2) \\
& z_{2} \rightarrow(1234) \\
& z_{3} \rightarrow(132) \\
& y_{4} \rightarrow(12) \\
& z_{4} \rightarrow(12)
\end{aligned}
$$

b) From the above permutation representations we deduce (see [14]), not only the inclusions of the above lemma, but the generators of each quadrangle group in terms of the generators of the corresponding triangle group which contains it; for instance, sets of left-coset representatives for the above inclusions are given by:

$$
\begin{gathered}
\left\{\Gamma_{1}, z_{1} \Gamma_{1}, z_{1}^{2} \Gamma_{1}, z_{1}^{3} \Gamma_{1}, z_{1}^{4} \Gamma_{1}, z_{1}^{5} \Gamma_{1}, z_{1}^{6} \Gamma_{1}\right\} ; \quad\left\{\Gamma_{2}, z_{2} \Gamma_{2}, z_{2}^{2} \Gamma_{2}, z_{2}^{3} \Gamma_{2}\right\} ; \\
\left\{\Gamma_{3}, y_{3} \Gamma_{3}, y_{3}^{2} \Gamma_{3}\right\} ; \quad\left\{\Gamma_{4}, y_{4} \Gamma_{4}\right\}
\end{gathered}
$$

where, of course, the notation we are considering is: $\Delta_{i}=\left\langle x_{i}, y_{i}, z_{i}\right| x_{i}^{r_{i}}=$ $\left.y_{i}^{l_{i}}=z_{i}^{m_{i}}=x_{i} y_{i} z_{i}=1\right\rangle\left(\right.$ For $i=1^{\prime}$ everything is analogous to $\left.i=1\right)$. 
Therefore,

$$
\Gamma_{4}=\left\langle x_{4}, y_{4}^{-1} x_{4} y_{4}, y_{4}^{2} \mid x_{4}^{2}=\left(y_{4}^{-1} x_{4} y_{4}\right)^{2}=\left(y_{4}^{2}\right)^{2}=\left(x_{4}^{-1} z_{4}^{-2} x_{4}\right)^{3}=1\right\rangle
$$

and

$\Gamma_{3}=\left\langle x_{3}, y_{3}^{-1} x_{3} y_{3}, y_{3}^{-2} x_{3} y_{3}^{2} \mid x_{3}^{2}=\left(y_{3}^{-1} x_{3} y_{3}\right)^{2}=\left(y_{3}^{-2} x_{3} y_{3}^{2}\right)^{2}=\left(x_{3}^{-1} z_{3}^{-3} x_{3}\right)^{3}=1\right\rangle$.

Let us now consider the automorphisms of $\Gamma_{3}$ and $\Gamma_{4}$ given by conjugation by $y_{3} \in \Delta_{3}$ and $y_{4} \in \Delta_{4}$, respectively; that is, $\eta_{j}(\gamma)=y_{j}^{-1} \gamma y_{j}$, for any $\gamma \in \Gamma_{j}$ ( $j=3$ and 4 ), and let us denote by $\tau_{\sigma}$ the automorphism of $S_{4}$ obtained by conjugation by any $\sigma \in S_{4}$. Then, we have

$$
\begin{aligned}
& \varphi_{1}^{1} \circ \eta_{4}\left(x_{4}\right)=\varphi_{1}^{1}\left(y_{4}^{-1} x_{4} y_{4}\right)=(12)=\varphi_{2}^{3}\left(x_{4}\right) \\
& \varphi_{1}^{1} \circ \eta_{4}\left(y_{4}^{-1} x_{4} y_{4}\right)=\varphi_{1}^{1}\left(y_{4}^{-2} x_{4} y_{4}^{2}\right)=(23)((12)(34))(23)=(13)(24)=\varphi_{2}^{3}\left(y_{4}^{-1} x_{4} y_{4}\right) \\
& \varphi_{1}^{1} \circ \eta_{4}\left(y_{4}^{2}\right)=\varphi_{1}^{1}\left(y_{4}^{2}\right)=(23)=\varphi_{2}^{3}\left(y_{4}^{2}\right),
\end{aligned}
$$

therefore

$$
\operatorname{Ker}\left(\varphi_{1}^{1}\right)=y_{4}^{-1}\left(\operatorname{Ker} \varphi_{2}^{3}\right) y_{4}
$$

Proceeding in an analogous way as before, we obtain that

$$
\begin{aligned}
& \operatorname{Ker}\left(\varphi_{1}^{2}\right)=y_{4}^{-1}\left(\operatorname{Ker} \varphi_{2}^{2}\right) y_{4}, \quad \operatorname{Ker}\left(\varphi_{1}^{3}\right)=y_{4}^{-1}\left(\operatorname{Ker} \varphi_{2}^{1}\right) y_{4}, \\
& \operatorname{Ker}\left(\varphi_{3}^{1}\right)=y_{4}^{-1}\left(\operatorname{Ker} \varphi_{3}^{2}\right) y_{4} \quad \text { and } \quad \operatorname{Ker}\left(\varphi_{3}^{3}\right)=y_{4}^{-1}\left(\operatorname{Ker} \varphi_{3}^{3}\right) y_{4}
\end{aligned}
$$

which proves the statement about case iv).

Working out case iii) in a similar way, one obtains that

$\operatorname{Ker}\left(\varphi_{1}^{1}\right)=y_{3}^{-1}\left(\operatorname{Ker}\left(\varphi_{3}^{1}\right)\right) y_{3}, \operatorname{Ker}\left(\varphi_{2}^{2}\right)=y_{3}^{-1}\left(\operatorname{Ker}\left(\varphi_{1}^{1}\right)\right) y_{3}, \operatorname{Ker}\left(\varphi_{3}^{1}\right)=y_{3}^{-1}\left(\operatorname{Ker}\left(\varphi_{2}^{2}\right)\right) y_{3}$

$\operatorname{Ker}\left(\varphi_{1}^{2}\right)=y_{3}^{-1}\left(\operatorname{Ker}\left(\varphi_{3}^{2}\right)\right) y_{3}, \operatorname{Ker}\left(\varphi_{2}^{1}\right)=y_{3}^{-1}\left(\operatorname{Ker}\left(\varphi_{1}^{2}\right)\right) y_{3}, \operatorname{Ker}\left(\varphi_{3}^{2}\right)=\mathrm{y}_{3}^{-1}\left(\operatorname{Ker}\left(\varphi_{2}^{1}\right)\right) \mathrm{y}_{3}$

$\operatorname{Ker}\left(\varphi_{1}^{3}\right)=y_{3}^{-1}\left(\operatorname{Ker}\left(\varphi_{3}^{3}\right)\right) y_{3}, \operatorname{Ker}\left(\varphi_{2}^{3}\right)=y_{3}^{-1}\left(\operatorname{Ker}\left(\varphi_{1}^{3}\right)\right) y_{3}, \operatorname{Ker}\left(\varphi_{3}^{3}\right)=y_{3}^{-1}\left(\operatorname{Ker}\left(\varphi_{2}^{3}\right)\right) y_{3}$

which concludes the proof.

From the choice of the epimorphisms in Lemma 3 one can also calculate the multiplier system, that is, the fixed point behaviour of a given automorphism of the constructed surface. Note that the multipliers depend not on the particular group element but only on its conjugacy class, c.f. [17]. One can easily calculate the following multipliers, giving the number of fixed points as the number of entries for each class and also the local action of the automorphism which of course can be described as multiplication by a suitable root of unity:

$$
\begin{array}{cccccc}
C & 1 A & 2 A & 2 B & 3 A & 4 A \\
& 0 & -1,-1,-1,-1 & -1,-1,-1,-1 & \zeta_{3}, \zeta_{3}^{2} & 0 .
\end{array}
$$


The multiplier system is the only data we need to produce the characters of the linear representation of the automorphism group $S_{4}$ acting on the holomorphic $q$-differentials $H^{0}\left(S_{t}, \Omega^{q}\right)$ by using the Eichler trace formula, c.f. [5]. We obtain the following character table, $\chi_{q}$ denotes the character on the holomorphic $q$-differentials, the last column gives the decomposition into the irreducible characters:

$$
\begin{array}{ccccccl}
C & 1 A & 2 A & 2 B & 3 A & 4 A & \\
& () & (12) & (12)(34) & (123) & (1234) & \\
\chi_{1} & 3 & -1 & -1 & 0 & 1 & \psi_{5} \\
\chi_{2} & 6 & 2 & 2 & 0 & 0 & \psi_{1}+\psi_{3}+\psi_{4} \\
\chi_{3} & 10 & -2 & -2 & 1 & 0 & \psi_{2}+\psi_{4}+2 \psi_{5} \\
\chi_{4} & 14 & 2 & 2 & -1 & 0 & \psi_{1}+2 \psi_{3}+2 \psi_{4}+3 \psi_{5} \\
\chi_{5} & 18 & -2 & -2 & 0 & 0 & \psi_{2}+\psi_{3}+2 \psi_{4}+3 \psi_{5} \\
\chi_{6} & 22 & 2 & 2 & 1 & 0 & 2 \psi_{1}+\psi_{2}+2 \psi_{3}+3 \psi_{4}+2 \psi_{5} .
\end{array}
$$

So far we did not use any property of a special surface $S_{t}$ but our arguments remain valid for all parameters $t$. The representation $\chi_{1}=\psi_{5}$ is of particular interest as it describes not only the representation of the surface automorphisms on the holomorphic 1-differentials but can also be interpreted to be the action of the automorphism group on the canonical model, c.f. [16]. This representation $\rho_{1}: S_{4} \longrightarrow G L_{3}(\mathbb{Z})$ is given by the following matrices

$$
\begin{gathered}
2 A \\
A_{1} \\
\left(\begin{array}{ccc}
-1 & 0 & 0 \\
0 & 0 & 1 \\
0 & 1 & 0
\end{array}\right) \quad\left(\begin{array}{ccc}
-1 & 0 & 0 \\
0 & -1 & 0 \\
0 & 0 & 1
\end{array}\right) \quad\left(\begin{array}{ccc}
0 & 0 & -1 \\
-1 & 0 & 0 \\
0 & 1 & 0
\end{array}\right)
\end{gathered}
$$

associate to a basis of holomorphic 1-differentials $x, y$ and $z$. For this basis we can evaluate the projective points belonging to fixed points of special automorphisms. We have already seen that any order three element has fixed points belonging to the multiplier $\zeta_{3}$ therefore not only the unique eigenspace generated by the eigenvector $\left[-\zeta_{3}, \zeta_{3}^{2}, 1\right]$ to the eigenvalue $\zeta_{3}$ of $A_{3}$, written of course in projective coordinates, is a fixed point in the canonical model, but also all of its orbit under $\rho_{1}\left(S_{4}\right)$, which has length 8. A similar argument for $A_{2}$ which has a fixed point belonging to the multiplier -1 gives in projective coordinates an eigenvector $[s, 1,0]$ with an unknown $s$, as we expect to obtain a one parameter family of Riemann surfaces. (Note that though $[1,0,0]$ is also an eigenvector of $A_{2}$ to the eigenvalue -1 it can not lie on the canonical model as it also is an eigenvector of $A_{1}$, representing an automorphism of $S_{t}$ known to have no fixed points in common with $A_{2}$ ). The orbit of this point under the automorphism group has length 12 . 
The canonical model of a non-hyperelliptic curve of genus 3 is known to be a smooth plane quartic. This derives from the facts that:

- The collection of 15 possible 4-differentials

$$
x^{4}, x^{3} y, x^{3} z, x^{2} y^{2}, x^{2} y z, x^{2} z^{2}, x y^{3}, x y^{2} z, x y z^{2}, x z^{3}, y^{4}, y^{3} z, y^{2} z^{2}, y z^{3}, z^{4}
$$

contains a basis of holomorphic 4-differentials (Theorem of Noether, c.f. [5]).

- The dimension of the space of holomorphic 4-differentials is

$$
\operatorname{dim} H^{0}\left(S_{t}, \Omega_{4}\right)=14 .
$$

Therefore there must exist a relation between these 15 holomorphic differentials and this turns out to be the canonical model. Note that the converse that every smooth plane quartic is a canonical model is also true. Knowing that the zero divisor of any holomorphic 4-differential has degree 16 it is impossible for a holomorphic 4-differential to vanish at the collection of 20 fixed points we found above. This is the key tool to calculate the smooth plane quartic to be

$$
x^{4}+y^{4}+z^{4}-\frac{s^{4}+1}{s^{2}}\left(x^{2} y^{2}+x^{2} z^{2}+y^{2} z^{2}\right)=0 .
$$

Choosing the parameter $t=-\left(s^{4}+1\right) / s^{2}$ we obtain the one parameter family as it is given in (3.1). For later use we want to calculate one representative of the remaining two orbits of the fixed points associate to the automorphisms of the class $2 A$. Again it has to be an eigenvector of the associate matrix to the eigenvalue -1 and again written in projective coordinates this is given as $[r,-1,1]$ (the eigenvector $[1,0,0]$ can be ruled out for the same reason as above). Using (3.1) we obtain:

$$
\begin{aligned}
& 0=r^{4}+2+t\left(2 r^{2}+1\right) \\
& r= \pm \sqrt{-t \pm \sqrt{t^{2}-t-2}} .
\end{aligned}
$$

In a next step we would like to calculate the triangle inclusions from Lemma 4 in terms of the parameter $t$ or to put it another way in terms of function field extensions. The knowledge of the coordinates of the fixed points of the automorphism group together with the invariant function will be the key tool to calculate the parameter $t$ for which a triangle inclusion can be obtained. We therefore need to calculate a function $f_{1}$ on $S_{t}$ which is invariant under the action of $S_{4}$ with degree 24. Again the character table on the holomorphic $q$-differentials is helpful. We see that there are two linearly independent 6 -differentials which are invariant under $S_{4}$ according 
to the multiplicity 2 of the principal representation in $\chi_{6}$. Their quotient is a meromorphic function of degree 24. We also observe that there is a 2-differential invariant under $S_{4}$ and risen to the third power we already found $\left(x^{2}+y^{2}+z^{2}\right)^{3}$ to be one of the 6-differentials we are looking for. The 3-differential $x y z$ is not quite invariant but belongs to $\psi_{2}$ therefore the square $(x y z)^{2}$ is invariant. Evaluation at the fixed points shows that they are linearly independent thus we have calculated $f_{1}$ to be

$$
f_{1}=\frac{\left(x^{2}+y^{2}+z^{2}\right)^{3}}{(x y z)^{2}}
$$

not depending on the parameter $t$. Choosing

$$
r_{1}:=\sqrt{-t+\sqrt{t^{2}-t-2}} \text { and } r_{2}:=\sqrt{-t-\sqrt{t^{2}-t-2}}
$$

and $s$ we find the representatives of the 4 orbits of fixed points and their images under $f_{1}$ on the Riemann sphere to be:

$$
\begin{aligned}
P_{1}:=\left[r_{1},-1,1\right] & \longmapsto Q_{1}=\frac{\left(r_{1}^{2}+2\right)^{3}}{r_{1}^{2}} \\
P_{2}:=\left[-\zeta_{3}, \zeta_{3}^{2}, 1\right] & \longmapsto Q_{2}=0 \\
P_{3}:=\left[r_{2},-1,1\right] & \longmapsto Q_{3}=\frac{\left(r_{2}^{2}+2\right)^{3}}{r_{2}^{2}} \\
P_{4}:=[s, 1,0] & \longmapsto Q_{4}=\infty .
\end{aligned}
$$

From this it is easy to deduce the following lemma:

Lemma 5. The previously defined points $P_{1}, P_{2}, P_{3}, P_{4}$ on $S_{t}$ can be mapped to arbitrary points $Q_{1}, Q_{2}, Q_{3}, Q_{4}$ resp. on the Riemann sphere by some Möbius transformation of $f_{1}$ if and only if the parameter $t$ satisfies the equation

$$
\frac{16 t^{3}+48 t^{2}-6 t-92}{27 t+54}=-\frac{\lambda_{1}^{2}+1}{\lambda_{1}}
$$

where $\lambda_{1}:=\lambda\left(Q_{1}, Q_{2}, Q_{3}, Q_{4}\right):=\frac{\left(Q_{1}-Q_{2}\right)\left(Q_{3}-Q_{4}\right)}{\left(Q_{2}-Q_{3}\right)\left(Q_{4}-Q_{1}\right)}$ is the cross ratio.

Proof. As we know the critical values of the function $f_{1}$ and the fact that the cross ratio is invariant under Möbius transformations we only need to compare the cross ratio of the critical values with $\lambda_{1}$

$$
\frac{\left(r_{1}^{2}+2\right)^{3}}{r_{1}^{2}} \frac{r_{2}^{2}}{\left(r_{2}^{2}+2\right)^{3}}=\lambda_{1} .
$$

Replacing $r_{1}$ and $r_{2}$ by the appropriate expression in $t$ the result is easily obtained. 
Remark 6. 1) For a choice of 4 generic points on the Riemann sphere we would expect 6 different values for the cross ratio $\lambda$ by permuting these points, namely

$$
\lambda, \lambda^{-1}, 1-\lambda,(1-\lambda)^{-1}, \lambda(\lambda-1)^{-1},(\lambda-1) \lambda^{-1} .
$$

This of course belongs to a representation of the group $S_{3}$ acting as Möbius transformations on the Riemann sphere. However the values $\lambda, \lambda^{-1}$ (and all the other values being inverse to each other) belong to the same values of $t$ as it can be seen in (3.2). Being a cubic equation in $t$ we obtain therefore for every generic choice of 4 points 9 different values of $t$. This, of course, coincides with the fact that in the generic situation we obtain 9 different surface kernels in a quadrangle group $[2,2,2,3]$.

2) There are some constellations of points where $\lambda\left(Q_{1}, Q_{2}, Q_{3}, Q_{4}\right)$ obtain fewer values by permuting these points. They belong to the fixed points of the representation of $S_{3}$ in the preceding remark. These are $\lambda=0, \infty$ which are mapped in (3.2) as $-\frac{\lambda^{2}+1}{\lambda}=\infty, \lambda=1,-1$ which lead to $-2,2$ in (3.2), $\lambda=2, \frac{1}{2}$ which goes to $-\frac{5}{2}$ and finally $\frac{1 \pm i \sqrt{3}}{2}$ which lead to -1 . Of course some of these values cannot be obtained by a collection of 4 pairwise distinct points on the Riemann sphere, namely $0,1, \infty$, so it is not astonishing at all that their image values $-2, \infty$ play a special role in (3.2), but also the other values are special.

3) The right hand side of the equation (3.2) can be thought to be a function of the parameter $t$ namely

$$
\beta(t):=\frac{16 t^{3}+48 t^{2}-6 t-92}{27 t+54}
$$

This function turns out to be (and has to be, c.f [19]) a Belyi function on the Riemann sphere, that is a function with at most three critical values. In fact its first derivative is

$$
\frac{d \beta}{d t}=\frac{(2 t+5)(t+1)^{2}}{(t+2)^{2}}
$$

so that ramification takes place in the critical points $-5 / 2,-1,-2, \infty$ which are mapped under $\beta$ to $2,-2, \infty$ and $\infty$ resp. The entire set of preimages of $2,-2, \infty$ is $\beta^{-1}(2)=\{2,-5 / 2\}, \beta^{-1}(-2)=\{-1\}$ and $\beta^{-1}(\infty)=\{-2, \infty\}$. It is only among these critical values $2,-2, \infty$ that we do not get three but less preimages under $\beta$ therefore it is an appropriate guess to expect among these values the parameters $t$ which correspond to the surface kernels lying inside $[2,4,6]$ and $[2,3,9]$. 
In order to obtain the parameter $t$ corresponding to the complete list of algebraic curves belonging to the family $\mathcal{F}_{1}$ for which their surface groups lie in the quadrangle groups $\Gamma_{i}$ inside the triangle groups listed in Lemma 4, let us observe that these Riemann surfaces admit a Belyi function $\left(\phi_{l}^{j i}\right.$ : $\left.\mathbb{H} / K_{l}^{j i} \rightarrow \mathbb{H} / \Delta_{i}\right)$ for which every point on the fiber of one critical value, for any of the three critical values $\left\{q_{k}\right\}_{k=1}^{3}$, has the same ramification order; let us call such a meromorphic function a uniform Belyi function.

Moreover, we need to obtain the function field extensions (up to field isomorphisms) corresponding to the natural quotient $\mathbb{H} / \Gamma_{i} \rightarrow \mathbb{H} / \Delta_{i}$, that is, $\mathbb{C}\left(h_{i}(z)\right) \hookrightarrow \mathbb{C}(z)$ or the meromorphic functions $h_{i}: \mathbb{C P}^{1} \rightarrow \mathbb{C P}^{1}$, using strongly the conditions imposed by the fact that $h_{i} \circ f_{1}$ has to be an uniform Belyi function, for every $i=1,1^{\prime}, 2,3$ and 4 .

This last consideration gives us a set of positive numbers

$$
\left(\nu_{1}^{1 i}, \ldots, \nu_{s_{1}}^{1 i} ; \nu_{1}^{2 i}, \ldots, \nu_{s_{2}}^{2 i} ; \nu_{1}^{3 i}, \ldots, \nu_{s_{3}}^{3 i}\right),
$$

with $\left\{\nu_{n}^{k i}\right\}$ being the ramification order of the points on the fiber of $q_{k}$ by $h_{i}$. Once we obtain the function field extensions (see [1]), as we can identify (up to composition with a Möbius transformation) the critical values of $f_{1}$ that we have already denoted by $Q_{1}, Q_{2}, Q_{3}, Q_{4}$ with the corresponding points on the fiber of $q_{k}$ by $h_{i}$, let us denote them by $R_{1}^{i}, R_{2}^{i}, R_{3}^{i}$ and $R_{4}^{i}$, we obtain the values of $t$ imposing that $\beta(t)$ is equal to

$$
-\left(\lambda^{i}+\frac{1}{\lambda^{i}}\right),-\left(1-\lambda^{i}+\frac{1}{1-\lambda^{i}}\right) \text { and }-\left(\frac{\lambda^{i}}{\lambda^{i}-1}+\frac{\lambda^{i}-1}{\lambda^{i}}\right)
$$

with $\lambda^{i}=\lambda\left(R_{1}^{i}, R_{2}^{i}, R_{3}^{i}, R_{4}^{i}\right)$; namely, following the order of the cases of triangle inclusions listed in part $a$ ) of Lemma 4 and with the notation described above,

Proposition 7. A Riemann surface $S_{t} \in \mathcal{F}_{1}$ has the normaliser of its surface group contained in a triangle group or, in other words, $S_{t}$ has a uniform Belyi function which factorizes through the quotient by the automorphism group if and only if $t$ is obtained from the data of one of the following cases:

Case i)

$$
\begin{aligned}
& h_{1}(z)=\left(z^{2}-1\right)^{3}(z-(7+\sqrt{-7}) / 4) \\
& \left(\nu_{1}^{11}, \nu_{2}^{11}, \nu_{3}^{11} ; \nu_{1}^{21}, \nu_{2}^{21}, \nu_{3}^{21}, \nu_{4}^{21}, \nu_{5}^{21} ; \nu_{1}^{31}\right)=(3,3,1 ; 2,2,1,1,1 ; 7) . \\
& R_{1}^{1}=-\frac{1}{7} \sqrt{-7}+\frac{1}{14} \sqrt{294+14 \sqrt{-7}}, R_{2}^{1}=-\frac{1}{7} \sqrt{-7}-\frac{1}{14} \sqrt{294+14 \sqrt{-7}} \\
& R_{3}^{1}=\frac{-5}{4}+\frac{3 \sqrt{-7}}{28} \quad \text { and } \quad R_{4}^{1}=\frac{7+\sqrt{-7}}{4} .
\end{aligned}
$$




$$
\begin{aligned}
& \beta(t)=-\left(\lambda^{1}+\frac{1}{\lambda^{1}}\right)=[(-648886-15148 \sqrt{-1} \sqrt{-147+56 \sqrt{7}}+ \\
& +8399 \sqrt{-7} \sqrt{-147+56 \sqrt{7}}+112636 \sqrt{7} \sqrt{-147+56 \sqrt{7}} \\
& +297563 \sqrt{-147-56 \sqrt{7}})(140 \sqrt{7} \sqrt{-147+56 \sqrt{7}}-469-128 \sqrt{7} \\
& +367 \sqrt{-147+56 \sqrt{7}})] / \\
& {[20412(-324443-120064 \sqrt{7}+112636 \sqrt{7} \sqrt{-147+56 \sqrt{7}}} \\
& +297563 \sqrt{-147+56 \sqrt{7}})] \\
& \beta(t)=-\left(1-\lambda^{1}+\frac{1}{1-\lambda^{1}}\right)=-8(1024 \sqrt{-147+56 \sqrt{7}} \sqrt{-7} \\
& +206588 \sqrt{7} \sqrt{-147+56 \sqrt{7}}-868056-120064 \sqrt{7} \\
& +34048 \sqrt{-1} \sqrt{-147+56 \sqrt{7}}-17577 \sqrt{-7}+548443 \sqrt{-147+56 \sqrt{7}}) \\
& (140 \sqrt{7} \sqrt{-147+56 \sqrt{7}}-256 \sqrt{7}+367 \sqrt{-147+56 \sqrt{7}} \\
& +4 \sqrt{-1} \sqrt{-147+56 \sqrt{7}}+19 \sqrt{-147+56 \sqrt{7}} \sqrt{-7}) / \\
& {[2401(140 \sqrt{7} \sqrt{-147+56 \sqrt{7}}-469-128 \sqrt{7}+367 \sqrt{-147+56 \sqrt{7}})]} \\
& \beta(t)=-\left(\frac{\lambda^{1}-1}{\lambda^{1}}+\frac{\lambda^{1}}{\lambda^{1}-1}\right)=[2(868056-120064 \sqrt{7} \\
& -49196 \sqrt{-1} \sqrt{-147+56 \sqrt{7}}+7375 \sqrt{-147+56 \sqrt{7}} \sqrt{-7} \\
& -93952 \sqrt{7} \sqrt{-147+56 \sqrt{7}}+17577 \sqrt{-7}-250880 \sqrt{-147+56 \sqrt{7}})] / \\
& {[(140 \sqrt{7} \sqrt{-147+56 \sqrt{7}}-256 \sqrt{7}-367 \sqrt{-147+56 \sqrt{7}}} \\
& +4 \sqrt{-1} \sqrt{-147+56 \sqrt{7}}-19 \sqrt{-147+56 \sqrt{7}} \sqrt{-7}) \\
& (4 \sqrt{-1} \sqrt{-147+56 \sqrt{7}}+19 \sqrt{-147+56 \sqrt{7}} \sqrt{-7}+469-128 \sqrt{7})] \text {. }
\end{aligned}
$$

Let us point out that this 3 degree equation on $t$ has $-\frac{3-3 \sqrt{-7}}{2}$ as a solution. 


\section{Case ii)}

$$
\begin{aligned}
& h_{1^{\prime}}(z)=\left(z^{2}-1\right)^{3}(z-(7-\sqrt{-7}) / 4) ; \\
& \left(\nu_{1}^{11^{\prime}}, \nu_{2}^{11^{\prime}}, \nu_{3}^{11^{\prime}} ; \nu_{1}^{21^{\prime}}, \nu_{2}^{21^{\prime}}, \nu_{3}^{21^{\prime}}, \nu_{4}^{31^{\prime}}, \nu_{5}^{31^{\prime}} ; \nu_{1}^{31^{\prime}}\right)=(3,3,1 ; 2,2,1,1,1 ; 7) . \\
& R_{1}^{\prime}=\frac{1}{7} \sqrt{-7}+\frac{1}{14} \sqrt{294+14 \sqrt{-7}}, \\
& R_{2}^{\prime}=\frac{1}{7} \sqrt{-7}-\frac{1}{14} \sqrt{294+14 \sqrt{-7}} \\
& R_{3}^{\prime}=\frac{-5}{4}-\frac{3 \sqrt{-7}}{28}
\end{aligned}
$$

and

$$
\begin{aligned}
R_{4}^{\prime}= & \frac{7-\sqrt{-7}}{4} . \\
\beta(t)= & -\left(\lambda^{1^{\prime}}+\frac{1}{\lambda^{1 \prime}}\right)=[(-648886+8399 \sqrt{-7} \sqrt{-147+56 \sqrt{7}} \\
& -15148 \sqrt{-1} \sqrt{-147+56 \sqrt{7}}+112636 \sqrt{7} \sqrt{-147+56 \sqrt{7}} \\
& +297563 \sqrt{-147+56 \sqrt{7}}) \\
& (-469+140 \sqrt{7} \sqrt{-147+56 \sqrt{7}}+367 \sqrt{-147+56 \sqrt{7}}-128 \sqrt{7}) \\
& (469+19 \sqrt{-7} \sqrt{-147+56 \sqrt{7}}+4 \sqrt{-1} \sqrt{-147+56 \sqrt{7}}-128 \sqrt{7})] \\
& {[20412(-324443+112636 \sqrt{7} \sqrt{-147+56 \sqrt{7}}} \\
& +297563 \sqrt{-147+56 \sqrt{7}}-120064 \sqrt{7})]
\end{aligned}
$$$$
\beta(t)=-\left(1-\lambda^{1^{\prime}}+\frac{1}{1-\lambda^{1^{\prime}}}\right)=-[(1024 \sqrt{-7} \sqrt{-147+56 \sqrt{7}}
$$$$
-206588 \sqrt{7} \sqrt{-147+56 \sqrt{7}}+868056-17577 \sqrt{-7}
$$$$
+34048 \sqrt{-1} \sqrt{-147+56 \sqrt{7}}
$$$$
+120064 \sqrt{7}-548443 \sqrt{-147+56 \sqrt{7}})(-140 \sqrt{7} \sqrt{-147+56 \sqrt{7}}
$$$$
-367 \sqrt{-147+56 \sqrt{7}}+256 \sqrt{7}+19 \sqrt{-7} \sqrt{-147+56 \sqrt{7}}+
$$$$
+4 \sqrt{-1} \sqrt{-147+56 \sqrt{7})}] /
$$$$
[-469+140 \sqrt{7} \sqrt{-147+56 \sqrt{7}}+367 \sqrt{-147+56 \sqrt{7}}-128 \sqrt{7}]
$$ 


$$
\begin{aligned}
\beta(t)= & -\left(\frac{\lambda^{1^{\prime}}-1}{\lambda^{1^{\prime}}}+\frac{\lambda^{1^{\prime}}}{\lambda^{1^{\prime}}-1}\right)=-2[7375 \sqrt{-7} \sqrt{-147+56 \sqrt{7}} \\
& -49196 \sqrt{-1} \sqrt{-147+56 \sqrt{7}}+120064 \sqrt{7}+93952 \sqrt{7} \sqrt{-147+56 \sqrt{7}} \\
& +250880 \sqrt{-147+56 \sqrt{7}}-868056++17577 \sqrt{-7}] / \\
& {[(-140 \sqrt{7} \sqrt{-147+56 \sqrt{7}}-367 \sqrt{-147+56 \sqrt{7}}+256 \sqrt{7}+} \\
& +19 \sqrt{-7} \sqrt{-147+56 \sqrt{7}}+4 \sqrt{-1} \sqrt{-147+56 \sqrt{7}})(-469 \\
& +19 \sqrt{-7} \sqrt{-147+56 \sqrt{7}}+4 \sqrt{-1} \sqrt{-147+56 \sqrt{7}}+128 \sqrt{7})] .
\end{aligned}
$$

Let us point out that this 3 degree equation on $t$ has $-\frac{3+3 \sqrt{-7}}{2}$ as a solution. Case iii)

$h_{2}(z)=z^{3}(1-z) ; \quad\left(\nu_{1}^{12}, \nu_{2}^{12}, \nu_{3}^{12} ; \nu_{1}^{22}, \nu_{2}^{22} ; \nu_{1}^{32}\right)=(2,1,1 ; 3,1 ; 4)$.

$R_{1}^{2}=\frac{-1+\sqrt{-2}}{4}, R_{2}^{2}=\infty, R_{3}^{2}=\frac{-1-\sqrt{-2}}{4}$ and $R_{4}^{2}=1$.

$\beta(t)=-\left(\lambda^{2}+\frac{1}{\lambda^{2}}\right)=-46 / 27$, with solutions $t=0$ and $\frac{-3 \pm \sqrt{-1}}{2}$.

$\beta(t)=-\left(1-\lambda^{2}+\frac{1}{1-\lambda^{2}}\right)=-\frac{35}{54}+\frac{95}{108} \sqrt{-2}$ and

$\beta(t)=-\left(\frac{\lambda^{2}-1}{\lambda^{2}}+\frac{\lambda^{2}}{\lambda^{2}-1}\right)=-\frac{35}{54}-\frac{95}{108} \sqrt{-2}$.

Case iv)

$h_{3}(z)=z^{3} ; \quad\left(\nu_{1}^{13}, \nu_{2}^{13}, \nu_{3}^{13} ; \nu_{1}^{23} ; \nu_{1}^{33}\right)=(1,1,1 ; 3 ; 3)$.

$R_{1}^{3}=1, R_{2}^{3}=\frac{-1+\sqrt{-3}}{2}, R_{3}^{3}=\frac{-1-\sqrt{-3}}{2}$ and $R_{4}^{3}=0$.

$\beta(t)=-\left(\lambda^{3}+\frac{1}{\lambda^{3}}\right)=-\left(1-\lambda^{3}+\frac{1}{1-\lambda^{3}}\right)=-\left(\frac{\lambda^{3}-1}{\lambda^{3}}+\frac{\lambda^{3}}{\lambda^{3}-1}\right)=-1 ;$

or, equivalently, the roots of $16 t^{3}+48 t^{2}+21 t-38$.

Case v)

$$
\begin{aligned}
& h_{4}(z)=z^{2} ; \quad\left(\nu_{1}^{14}, \nu_{2}^{14} ; \nu_{1}^{24} ; \nu_{1}^{34}\right)=(1,1 ; 2 ; 2) . \\
& R_{1}^{4}=0, R_{2}^{4}=1, R_{3}^{4}=-1 \text { and } R_{4}^{4}=\infty . \\
& \beta(t)=-\left(\lambda^{4}+\frac{1}{\lambda^{4}}\right)=-\left(\frac{\lambda^{4}-1}{\lambda^{4}}+\frac{\lambda^{4}}{\lambda^{4}-1}\right)=2 ; \\
& \beta(t)=-\left(1-\lambda^{4}+\frac{1}{1-\lambda^{4}}\right)=-\frac{5}{2} .
\end{aligned}
$$


Proposition 8. For every $t$ and $t^{\prime} \in \mathbb{C}-\{-1, \pm 2,-3(1 \pm \sqrt{-7}) / 2\}$, the genus 3 compact Riemann surfaces $S_{t}$ and $S_{t^{\prime}}$ in $\mathcal{F}_{1}$ are isomorphic if and only if $t=t^{\prime}$ and for $t=-3(1 \pm \sqrt{-7}) / 2$ both surfaces are isomorphic.

Proof. $S_{t} \cong S_{t^{\prime}}$ but $S_{t} / S_{4} ¥ S_{t^{\prime}} / S_{4}$ implies that there exist two non-conjugate subgroups of Aut $\left(S_{t}\right)$ isomorphic to $S_{4}$ and, in particular, Aut $\left(S_{t}\right)>S_{4}$. In terms of Fuchsian groups, if we denote by $K$ the surface group of $S_{t}$, then the above statement is equivalent to the existence of two Fuchsian groups $\Gamma$ and $\Gamma^{\prime}$ with signature $[2,2,2,3]$, included and non-conjugate in $N(K)$, and such that $K \unlhd \Gamma$ and $K \unlhd \Gamma^{\prime}$. It was proved in Lemma 4 that the previous statement is only possible for $N(K)=[2,3,7]=\Delta_{1}$; in fact, both quadrangle groups $\Gamma_{1}$ and $\Gamma_{1^{\prime}}$ contain the surface group of the Klein's quartic for which the values of $t$ as a member of the family $\mathcal{F}_{1}$ are $\frac{-3(1 \pm \sqrt{-7})}{2}$ (see [12] and parts $i$ ) and $i^{\prime}$ ) of the above proposition), so $S_{t}$ and $S_{t^{\prime}}$ in $\mathcal{F}_{1}$ are isomorphic surfaces only if $S_{t} / S_{4} \cong S_{t^{\prime}} / S_{4}$ unless $t=\frac{-3(1 \pm \sqrt{-7})}{2}$.

On the other hand, if we consider the Möbius transformation interchanging the two critical values $\frac{\left(r_{1}^{2}+2\right)^{3}}{r_{1}^{2}}$ and $\frac{\left(r_{2}^{2}+2\right)^{3}}{r_{2}^{2}}$, we obtain that there exists an element of $S_{4}$ that acts by conjugation as an automorphism of $S_{4}$ and makes commutative the associated diagram as in Lemma 1 , with $\eta$ realizes by conjugation by an element of $\Gamma$, that is, belonging to the normalizer of $K$ so it lifts to an automorphism of $S_{t}$. Moreover, if we fix the cross ratio $\lambda$ of the four critical values of the morphism $f_{1}$, for any of the nine epimorphisms of Lemma 3 , we obtain three values of $t$, namely the solutions of the equation:

$$
16 t^{3}+48 t^{2}-6 t-92+(27 t+54)\left(\lambda+\frac{1}{\lambda}\right)=0 .
$$

If we consider the order 3 Möbius transformations permuting the three critical values with ramification order equals 2 , we obtain the nine non-equivalent epimorphisms that, as was proved in Lemma 3, they are not equivalent or, in other words, they do not differ by post-composition with an automorphism of $S_{4}$ therefore, according to Lemma 1, they do not lift to isomorphisms of $S_{t}$ which finishes the proof.

Moreover,

Theorem 9. Let $\left[S_{t}\right]$ be the isomorphism class of any genus 3 compact Riemann surface belonging to the family $\mathcal{F}_{1}$. Then

$$
\Phi: t \mapsto\left[S_{t}\right]
$$

is a closed and injective, outside a proper subvariety, mapping between the analytic space $\mathbb{C}-\{ \pm 2,-1\}$ and its image inside the moduli space $\mathcal{M}_{3}$ of compact Riemann surfaces of genus 3 ; more precisely, $\mathbb{C}-\{ \pm 2,-1\}$ is the normalization of its image which is the irreducible subvariety of $\mathcal{M}_{3}$ consisting of those surfaces admitting an automorphism group isomorphic to $S_{4}$. 
Proof. The theorem follows from the proof of Proposition 8, [7] and as a similar example to the one exposed in Section 5.4.9 in [11] to the Theorem in Section 5.9.3 in [11], highlighting the canonical nature of Teichmüller space and its natural complex structure concerning marked families of compact Riemann surfaces with distinguished points and the Universal Property.

It is a classical result that the Weierstrass points of a genus $g$ compact Riemann surface are given by the zeros of a $\frac{g(g+1)}{2}$-holomorphic differential named the Wronskian of a suitable basis. For every genus 3 curve $S_{t}$ belonging to the family $\mathcal{F}_{1}$ we obtain the 6 -holomorphic differential which is the Wronskian $\left(\mathrm{Wr}_{1}\left(S_{t}\right)\right)$ of a basis for the vector space of holomorphic differentials given by $x, y$ and $z$. In order to make the direct computation which is the Wronskian for this basis, that is, the determinant of the $3 \times 3$ matrix composed of the $(j-1)$-derivatives of (the local expressions of) each element of this basis at the $j$ row, we use the meromorphic function $x / z$ as a local parameter at every point of the curve, unless a finite number of them, in the affine neighborhood $z=1$ and, finally, we return it to a global expression what gives us that

Theorem 10. For every compact Riemann surface $S_{t}$ belonging to the family $\mathcal{F}_{1}$, the Wronskian of the basis $\{x, y, z\}$ for the space of holomorphic differentials is

$$
\begin{array}{r}
\mathrm{Wr}_{1}\left(S_{t}\right)=\left(x^{4} y^{2}+2 x^{2} y^{2} z^{2}+y^{2} z^{4}+x^{4} z^{2}+x^{2} z^{4}\right) t^{2} \\
+\left(z^{6}+x^{6}-x^{4} y^{2}-y^{2} z^{4}\right) t-6 x^{2} y^{2} z^{2}
\end{array}
$$

\section{Case (II). Moduli of the family $\mathcal{F}_{2}$}

For every $t \in \mathbb{C}-\{ \pm 2\}$, the smooth projective curve described by A. Kuribayashi and K. Komiya in [10] as a member of the family $\mathcal{F}_{2}$ was given by:

$$
S_{t} \equiv\left\{y^{4}=x^{4}-t x^{2} z^{2}+z^{4}\right\} \subseteq \mathbb{C P}^{2} .
$$

This is a canonical model for the associated genus 3 compact Riemann surface with automorphism group $G_{2}$, being

$$
\begin{aligned}
G_{2} & =<\left\{\alpha, \beta, \sigma: \alpha^{2}=\beta^{2}=\sigma^{4}=1 ; \alpha \sigma=\sigma \alpha ; \beta \sigma=\sigma \beta ; \beta \alpha=\alpha \beta \sigma^{2}\right\}> \\
& \cong \mathrm{C}_{2} \ltimes\left(\mathrm{C}_{2} \times \mathrm{C}_{4}\right) \quad \text { where } \operatorname{Center}\left(\mathrm{C}_{2} \ltimes\left(\mathrm{C}_{2} \times \mathrm{C}_{4}\right)\right)=\mathrm{C}_{4} .
\end{aligned}
$$

We will proceed as in the previous section and in order to calculate the number of different epimorphisms $\varphi$ from

$$
\Gamma=\left\langle x, y, z \mid x^{2}=y^{2}=z^{2}=(x y z)^{-4}=1\right\rangle
$$


Fuchsian quadrangle group with signature $[2,2,2,4]$ to the automorphism group $G_{2}$, the character table of $\mathrm{C}_{2} \ltimes\left(\mathrm{C}_{2} \times \mathrm{C}_{4}\right)$ with $\operatorname{Center}\left(\mathrm{C}_{2} \ltimes\left(\mathrm{C}_{2} \times\right.\right.$ $\left.\left.\mathrm{C}_{4}\right)\right)=\mathrm{C}_{4}$ is helpful

$\begin{array}{ccccccccccc}C & 1 A & 2 A & 2 B & 2 D & 2 E & 4 A_{1} & 4 A_{2} & 4 A_{3} & 4 A_{4} & 4 A_{5} \\ & \text { Id } & \sigma^{2} & \beta & \alpha & \alpha \beta \sigma & \sigma & \sigma^{3} & \beta \sigma & \alpha \sigma & \alpha \beta \\ C \mid & 1 & 1 & 2 & 2 & 2 & 1 & 1 & 2 & 2 & 2 \\ \psi_{1} & 1 & 1 & 1 & 1 & 1 & 1 & 1 & 1 & 1 & 1 \\ \psi_{2} & 1 & 1 & 1 & -1 & -1 & 1 & 1 & 1 & -1 & -1 \\ \psi_{3} & 1 & 1 & -1 & 1 & -1 & 1 & 1 & -1 & 1 & -1 \\ \psi_{4} & 1 & 1 & -1 & -1 & 1 & 1 & 1 & -1 & -1 & 1 \\ \psi_{5} & 1 & 1 & 1 & 1 & -1 & -1 & -1 & -1 & -1 & 1 \\ \psi_{6} & 1 & 1 & 1 & -1 & 1 & -1 & -1 & -1 & 1 & -1 \\ \psi_{7} & 1 & 1 & -1 & 1 & 1 & -1 & -1 & 1 & -1 & -1 \\ \psi_{8} & 1 & 1 & -1 & -1 & -1 & -1 & -1 & 1 & 1 & 1 \\ \psi_{9} & 2 & -2 & 0 & 0 & 0 & 2 i & -2 i & 0 & 0 & 0 \\ \psi_{10} & 2 & -2 & 0 & 0 & 0 & -2 i & 2 i & 0 & 0 & 0 .\end{array}$

It is easy to check that in order to get an epimorphism from $\Gamma$ to $G_{2}$ we need to apply the three order two generators of $\Gamma$ to each of the three conjugacy classes of non-central involutions. Thus the number of different epimorphisms equals the number of solutions of the equations $\bar{x}_{1}^{2}=\bar{x}_{2}^{2}=$ $\bar{x}_{3}^{2}=\bar{x}_{4}^{4}=\bar{x}_{1} \bar{x}_{2} \bar{x}_{3} \bar{x}_{4}=1$, where $\bar{x}_{1}, \bar{x}_{2}$ and $\bar{x}_{3}$ belong to any permutation of the conjugacy classes $2 B, 2 D$ and $2 E$, and $\bar{x}_{4}$ belongs to $4 A_{1}, 4 A_{2}, 4 A_{3}, 4 A_{4}$ or $4 A_{5}$. This number $n_{\varphi}$ can be easily calculated by using the well known formula quoted in the previous section,

$$
n_{\varphi}:=\sum_{j=1}^{5} 6 \frac{|2 B||| 2 D|| 2 E|| 4 A_{j} \mid}{\left|G_{2}\right|} \sum_{i=1}^{10} \frac{\psi_{i}\left(\bar{x}_{1}\right) \psi_{i}\left(\bar{x}_{2}\right) \psi_{i}\left(\bar{x}_{3}\right) \psi_{i}\left(\bar{x}_{4}\right)}{\psi_{i}(1)^{2}}=48
$$

Of course to obtain the number of different surface kernels we have to bring into account that two chosen image quadruples lead to the same surface kernel if and only if they differ by an automorphism of the group $G_{2}$. The automorphism group of $G_{2}$ has order $48=2 \times 6 \times 4$. The possible images by an automorphism of $\sigma$ are $\sigma$ and $\sigma^{3}$. And, if we denote by $\mu$ the image of one of the order two generators $\alpha$ and $\beta$ among any of the six non-central involutions, the other one only can not be applied to $\mu$ or $\mu \sigma^{2}$. Therefore we obtain the total number of different kernels inside a $[2,2,2,4]$ quadrangle group to be $n_{\varphi} / 48=1$. It is obvious that this epimorphism can be given in the following way: 
Lemma 11. For every Fuchsian group $\Gamma$ with signature $[2,2,2,4]$, there exists a unique non-equivalent surface kernel epimorphism $\varphi: \Gamma \rightarrow G_{2}$ given by

$$
\varphi(x)=\alpha, \quad \varphi(y)=\beta \quad \text { and } \varphi(z)=\alpha \beta \sigma .
$$

As was exposed in the previous section and with a similar notation, from the choice of the epimorphism in Lemma 11 one can calculate the multiplier system:

$$
\begin{aligned}
& \begin{array}{lllllr}
C & 1 A & 2 A & 2 B & 2 D & 2 E
\end{array} \\
& 0 \quad-1,-1,-1,-1 \quad-1,-1,-1,-1 \quad-1,-1,-1,-1 \quad-1,-1,-1,-1 \\
& \begin{array}{lllll}
4 A_{1} & 4 A_{2} & 4 A_{3} & 4 A_{4} & 4 A_{5}
\end{array} \\
& \begin{array}{lllll}
i, i, i, i & i, i, i, i & 0 & 0 & 0 .
\end{array}
\end{aligned}
$$

As we did in the previous section, knowing the multiplier system we obtain via Eichler trace formula the characters of the linear representations of the automorphism group $G_{2}$ acting on the holomorphic $q$-differentials:

$\begin{array}{ccccccccccc}C & 1 A & 2 A & 2 B & 2 D & 2 E & 4 A_{1} & 4 A_{2} & 4 A_{3} & 4 A_{4} & 4 A_{5} \\ & \mathrm{Id} & \sigma^{2} & \beta & \alpha & \alpha \beta \sigma & \sigma & \sigma^{3} & \beta \sigma & \alpha \sigma & \alpha \beta \\ |C| & 1 & 1 & 2 & 2 & 2 & 1 & 1 & 2 & 2 & 2 \\ \chi_{1} & 3 & -1 & -1 & -1 & -1 & -1+2 i & -1-2 i & 1 & 1 & 1 \\ \chi_{2} & 6 & 2 & 2 & 2 & 2 & -2-2 i & -2+2 i & 0 & 0 & 0 \\ \chi_{3} & 10 & -2 & -2 & -2 & -2 & 2-2 i & 2+2 i & 0 & 0 & 0 \\ \chi_{4} & 14 & 2 & 2 & 2 & 2 & 2+2 i & 2-2 i & 0 & 0 & 0 .\end{array}$

Therefore, the decomposition into irreducible characters is:

$$
\begin{aligned}
& \chi_{1}=\psi_{8}+\psi_{9} \\
& \chi_{2}=\psi_{1}+\psi_{5}+\psi_{6}+\psi_{7}+\psi_{10} \\
& \chi_{3}=2 \psi_{10}+\psi_{9}+\psi_{8}+\psi_{2}+\psi_{3}+\psi_{4} \\
& \chi_{4}=2 \psi_{1}+\psi_{7}+\psi_{6}+\psi_{5}+\psi_{4}+\psi_{3}+\psi_{2}+2 \psi_{9}+\psi_{10} .
\end{aligned}
$$

Again, for any surface $S_{t} \in \mathcal{F}_{2}$, the representation $\chi_{1}=\psi_{8}+\psi_{9}$ can also be thought as the action of the automorphism group on the canonical model. This representation $\rho_{2}: G_{2} \rightarrow G L_{3}(\mathbb{C})$ is given by the following matrices

$$
\begin{aligned}
& \begin{array}{lll}
2 B & 2 D & 4 A_{1}
\end{array} \\
& B_{1} \quad B_{2} \quad B_{3} \\
& \left(\begin{array}{ccc}
1 & 0 & 0 \\
0 & -1 & 0 \\
0 & 0 & -1
\end{array}\right) \quad\left(\begin{array}{ccc}
0 & 0 & -1 \\
0 & -1 & 0 \\
-1 & 0 & 0
\end{array}\right) \quad\left(\begin{array}{ccc}
i & 0 & 0 \\
0 & -1 & 0 \\
0 & 0 & i
\end{array}\right)
\end{aligned}
$$

associate to a basis of holomorphic 1-differentials $x, y$ and $z$. 
Using the same procedure as in the first section, we can obtain the canonical model for each member of the family $\mathcal{F}_{2}$ as the linear combination of the 15 possible 4 -differentials

$y^{4}, y^{3} z, y^{3} x, y^{2} z^{2}, y^{2} z x, y^{2} x^{2}, y z^{3}, y z^{2} x, y z x^{2}, y x^{3}, z^{4}, z^{3} x, z^{2} x^{2}, z x^{3}, x^{4}$

which vanishes at the collection of the following 20 points given in projective coordinates: the fixed point of $B_{1}[0, u, 1]$, obtained as one of its eigenvectors associated to the eigenvalue -1 , and the 8 points on its orbit under the action of $\rho_{2}\left(G_{2}\right)$; in a similar way, the fixed point of $B_{2}[1, s, 1]$ and the 8 points on its orbit; and, finally, the fixed point of $B_{3},[w, 0,1]$, eigenvector associated to the eigenvalue $i$ and, of course, the 4 points on its orbit. The resulting smooth plane quartic is:

$$
\left(x^{\prime}\right)^{4}+\frac{r^{2} s^{4}}{\left(r^{2}-1\right)^{2}}\left(y^{\prime}\right)^{4}-\frac{\left(1+r^{4}\right) s^{4}}{\left(r^{2}-1\right)^{2}}\left(y^{\prime}\right)^{2}\left(z^{\prime}\right)^{2}+\frac{r^{2} s^{4}}{\left(r^{2}-1\right)^{2}}\left(z^{\prime}\right)^{4}=0 .
$$

After the change of projective coordinates given by

$$
x=x^{\prime}, \quad y=s \sqrt{\frac{r}{r^{2}-1}} y^{\prime}, \quad z=s \sqrt{\frac{r}{r^{2}-1}} z^{\prime}
$$

and choosing the parameter $t=\frac{1+r^{4}}{r^{2}}$, we obtain the one parameter family as it was given in (4.1).

In this section, the invariant 16 degree meromorphic function $f_{2}$ on $S_{t} \in$ $\mathcal{F}_{2}$ can be obtained as the quotient of two linear independent 4-differentials, according to the multiplicity 2 of the principal representation in $\chi_{4}$. Evaluation at the fixed points shows that the holomorphic 4-differentials $x^{4}+z^{4}$ and $x^{2} z^{2}$ are linear independent and, as they are obviously invariant under the action of $G_{2}$, we have calculated $f_{2}$ to be

$$
f_{2}=\frac{x^{4}+z^{4}}{x^{2} z^{2}}
$$

By other hand, now using (4.1) we can get a representative for each of the 4 orbits of fixed points and their images under $f_{2}$ on the Riemann sphere, which are

$$
\begin{aligned}
P_{1}:=[1, s, 1] & \longmapsto Q_{1}=2 \\
P_{2}:=[0, u, 1] & \longmapsto Q_{2}=\infty \\
P_{3}:=[-i, v, 1] & \longmapsto Q_{3}=-2 \\
P_{4}:=[w, 0,1] & \longmapsto Q_{4}=t,
\end{aligned}
$$

where $u^{4}=1, s^{4}=2-t, v^{4}=2+t$ and $w^{4}-t w^{2}+1=0$. 
Lemma 12. There exists a subgroup of automorphisms of $G_{2}$ which permutes the conjugacy classes of $\alpha, \beta$ and $\alpha \beta \sigma$ as $S_{3}$.

Proof. Let us denote by $H$ and $T \in \operatorname{Aut}\left(G_{2}\right)$ the automorphisms of $G_{2}$ described by:

$$
\begin{gathered}
H(\alpha)=\beta, \quad H(\beta)=\alpha \beta \sigma, \quad H(\sigma)=\sigma . \\
T(\alpha)=\beta, \quad T(\beta)=\alpha, \quad T(\sigma)=\sigma^{3} .
\end{gathered}
$$

The subgroup of $\operatorname{Aut}\left(G_{2}\right)$ generated by $H$ and $T$ satisfies the required conditions.

Lemma 13. There exist the following quadrangle groups $\Gamma_{i}$ with signature $[2,2,2,4]$ included and non-conjugate in a bigger Fuchsian group $\Delta_{i}$ :
i) $\Gamma_{1}=[2,2,2,4]_{1}$
ii) $\Gamma_{2}=[2,2,2,4]_{2}$
$\leq[2,4,5]=\Delta_{1}$ with index 5
iii) $\Gamma_{3}=[2,2,2,4]_{3}$
iv) $\Gamma_{4}=[2,2,2,4]_{4}$

with indexes 2 and 3 , respectively.

Proof. Using the simple area argument $\left[\Delta_{i}: \Gamma_{i}\right]=N=\mu\left(\Gamma_{i}\right) / \mu\left(\Delta_{i}\right)$ and $\mu([2,2,2,4])=1 / 4$, for any possible inclusion $\Gamma_{i} \leq \Delta_{i}$ with $\Gamma_{i}$ a Fuchsian group of signature $[2,2,2,4]$ we must have $\mu\left(\Delta_{i}\right)=1 /(4 N)$ with $N \geq 2$. On the other hand, as for any $4 \leq m \leq l$ we have $\mu([3, m, l]) \geq \mu([3,4,4])=$ $1 / 6>1 / 8=1 /(2 N)$ and, for any $6 \leq n \leq s, \mu([2, n, s]) \geq \mu([2,6,6])=$ $1 / 6>1 / 8$, we only need to check if it is possible that there exist such inclusions for $\Delta_{i}$ with signatures $[3,3,4]$ and $[2, n, l]$, for $l \geq n$ and $n=3,4$ and 5. Moreover, it was proved in Theorem 1 in [14] that the existence of an inclusion of Fuchsian groups of a given index $N$ is equivalent to the existence of a permutation representation inside the symmetric group of degree $N, S_{N}$, satisfying certain conditions. In particular, as the elliptic elements of the subgroup have to be conjugated to powers of the elliptic elements of the bigger group, then the orders of these ones have to divide the orders of those ones. Hence, first of all, let us study the last case for $n=3,4$ and 5, independently:

- $\mu([2,3, l])=\frac{1}{6}-\frac{1}{l}=\frac{l-6}{6 l}=1 /(4 N)$, which implies that $(l-6)$ divides $3 l$. Then, $l-6$ has to divide as well $3 l-3(l-6)=18$. Therefore, we have the possibilities $l=7,8,9,12,15$ and 24 . But only $l=8$ and 12 are really possible as they are the unique multiples of 4 ; in fact, both triangle groups contain a subgroup of signature $[2,2,2,4]$ as we will show afterwards giving the corresponding permutation representations, according to the mentioned Theorem 1 in [14]. 
- $\mu([2,4, l])=1 / 4-1 / l=(l-4) /(4 l)=1 /(4 N)$ which implies that $l-4$ divides $l$, hence $l-4$ has to divide $l+(-1) \cdot(l-4)=4$. Then, the unique possibilities are $l=5,6$ and 8 . We will show as well that all of these possible inclusions really happen.

- $\mu([2,5, l])=3 /(10)-1 / l=(3 l-10) /(10 l)$. Again, this implies that $3 l-10$ divides $100=3 \cdot(10 l)+(-10) \cdot(3 l-10)$. With this in mind and the fact that $l$ has to be an integer multiple of 4 , we obtain as unique possibility $l=4$ which was already included in the preceding case.

Summarizing, the unique Fuchsian groups containing a quadrangle group with signature $[2,2,2,4]$ are the ones exposed in this lemma because, according to Theorem 1 in [14], the existence of the above inclusions of Fuchsian groups comes from the existence of the following permutation representations $\theta_{i}$ of the action of the generators of the corresponding triangle group, $\Delta_{i}$, on the set of left coset representatives of $\Delta_{i} / \Gamma_{i}$, for $i=1,2,3$ and 4 ; more explicitly,

$$
\begin{aligned}
& \theta_{1}:[2,4,5] \rightarrow S_{5} \\
& x_{1} \rightarrow(1235)(4) \\
& y_{1} \rightarrow(1)(2)(3)(45) \\
& z_{1} \rightarrow(12345) \\
& \theta_{2}:[2,4,6] \rightarrow S_{3} \\
& x_{2} \rightarrow(1)(23) \\
& y_{2} \rightarrow(3)(12) \\
& z_{2} \rightarrow(123) \\
& \theta_{3}:[2,3,12] \rightarrow S_{3} \\
& x_{3} \rightarrow(1)(2)(3) \\
& \theta_{4}:[2,4,8] \rightarrow S_{2} \\
& x_{4} \rightarrow(1)(2) \\
& \theta_{4}^{\prime}:[2,3,8] \rightarrow S_{3} \\
& y_{3} \rightarrow(123) \\
& y_{4} \rightarrow(12) \\
& x_{4}^{\prime} \rightarrow(13)(2) \\
& z_{3} \rightarrow(132) \\
& z_{4} \rightarrow(12) \\
& y_{4}^{\prime} \rightarrow(123) \\
& z_{4}^{\prime} \rightarrow(12)(3) \text {. }
\end{aligned}
$$

Finally, we only can get one elliptic generator of order 2 from $[3,3,4]$ so this triangle group can not contain a group with signature $[2,2,2,4]$.

Proposition 14. $S_{t}$ and $S_{t^{\prime}}$ belonging to $\mathcal{F}_{2}$ are isomorphic if and only if $p_{2}\left(t^{\prime}\right)=p_{2}(t)$, where $p_{2}: \mathbb{C P}^{1} \rightarrow \mathbb{C P}^{1}$ is given by $p_{2}(z)=-16 \frac{z^{2}\left(z^{2}-36\right)^{2}}{\left(z^{2}-4\right)^{2}}$.

Proof. The proof follows from two steps:

i) As in the previous section, $S_{t} \cong S_{t^{\prime}}$ but $S_{t} / G_{2} ¥ S_{t^{\prime}} / G_{2}$ implies that there exist two non-conjugate subgroups of Aut $\left(S_{t}\right)$ isomorphic to $G_{2}$ and, in particular, Aut $\left(S_{t}\right)>G_{2}$. Therefore, if we denote by $K$ the surface group of $S_{t}$, in terms of Fuchsian groups the above statement is equivalent to the existence of two Fuchsian groups $\Gamma$ and $\Gamma^{\prime}$ with signature $[2,2,2,4]$, included and non-conjugate in $N(K)$, such that $K \unlhd \Gamma$ and $K \unlhd \Gamma^{\prime}$. On the other hand, it was proved in Lemma 13 that this never happens and we conclude that $S_{t} \cong S_{t^{\prime}}$ only if $S_{t} / G_{2} \cong S_{t^{\prime}} / G_{2}$. 
Let us observe that the uniqueness of the index 16 normal surface group inside any Fuchsian group with signature $[2,2,2,4]$, provided by Lemma 11, is enough to ensure that for the normal inclusions of cases iii) and iv) in Lemma 13 the corresponding genus 3 compact Riemann surfaces admit automorphism groups of orders 3 and, at least, 2 times the order of $G_{2}$, respectively.

ii) Combining Lemma 1 and Lemma 12, it is clear that the group isomorphic to $S_{3}$ of conformal automorphisms of $\mathbb{C P}^{1}$ permuting the three critical values of the covering $f_{2}: S_{t} \rightarrow S_{t} / G_{2}$ with branching orders 2 , lifts to isomorphisms of $S_{t}$. Now, the statement of proposition follows from $i$ ) and the fact that the cross ratios are:

$$
\begin{gathered}
\lambda_{2}=\lambda\left(Q_{1}, Q_{2}, Q_{3}, Q_{4}\right)=\lambda(2, \infty,-2, t)=\frac{t+2}{t-2} ; \lambda_{2}=\frac{t-2}{t+2}=\frac{1}{\frac{t+2}{t-2}} ; \\
\lambda_{2}=\frac{4}{2-t}=1-\frac{t+2}{t-2}, \quad \lambda_{2}=\frac{2-t}{4}=\frac{1}{1-\frac{t+2}{t-2}} \\
\lambda_{2}=\frac{4}{t+2}=\frac{\frac{t+2}{t-2}-1}{\frac{t+2}{t-2}} \quad \text { and } \quad \lambda_{2}=\frac{t+2}{4}=\frac{\frac{t+2}{t-2}}{\frac{t+2}{t-2}-1} .
\end{gathered}
$$

If we express $t$ in terms of $\lambda_{2}$ for each of the six cross ratios, the solutions are the images of $t$ under the action of the finite group of Möbius transformations, $Q_{2}$, isomorphic to $S_{3}$ and generated by

$$
S(z)=-z \quad \text { and } \quad N(z)=\frac{2(z+6)}{2-z} ;
$$

being the orbit of $t$ under $\langle N\rangle$ the solutions of $\lambda_{2}=\frac{t+2}{t-2}=\lambda(-2, t, 2, \infty)$, $\lambda_{2}=\frac{4}{t+2}=\lambda(2,-2, t, \infty)$ and $\lambda_{2}=\frac{2-t}{4}=\lambda(t, 2,-2, \infty)$. On the other hand, $p_{2}$ is the meromorphic function corresponding to the normal or Galoisian covering with covering group $Q_{2}$ because $\operatorname{deg}\left(p_{2}\right)=6=\operatorname{ord}\left(Q_{2}\right)$ and, for every $M \in Q_{2}$, we have that $p_{2} \circ M=p_{2}$.

We could say that $p_{2}$ is the analogous to the famous modular $j$-invariant for elliptic curves written as an invariant (degree 6) rational function of the classical Legendre modular function.

Theorem 15. Let $Q_{2}$ be the group of Möbius transformations isomorphic to $S_{3}$ generated by $z \mapsto-z$ and $z \mapsto \frac{2(z+6)}{2-z}$, and let $\left[S_{t}\right]$ be the isomorphism class of any genus 3 compact Riemann surface belonging to the family $\mathcal{F}_{2}$. Then the mapping

$$
\Phi: t \mapsto\left[S_{t}\right]
$$

is a holomorphic injection between the analytic space $(\mathbb{C}-\{ \pm 2\}) / Q_{2}$ and the moduli space $\mathcal{M}_{3}$ of compact Riemann surfaces of genus 3. Its image is the subvariety of $\mathcal{M}_{3}$ consisting of those surfaces admitting an automorphism group isomorphic to $\mathrm{C}_{2} \ltimes\left(\mathrm{C}_{2} \times \mathrm{C}_{4}\right)$ with center isomorphic to $\mathrm{C}_{4}$.

Proof. As in the previous section it follows from [11] and Proposition 14. 
Remark 16. $p_{2}: \mathbb{C P}^{1} \rightarrow \mathbb{C P}^{1}$ is a Belyi function with critical values 0,1728 and $\infty$. Note that the curves corresponding to the ramified points on the fiber of each critical value of $p_{2}$ are:

i) $p_{2}^{-1}(\infty)=\{\infty, \pm 2\}$, all of them with ramification order 2 . The algebraic equations in $\mathcal{F}_{2}$ corresponding to $t= \pm 2$ are reducible; namely:

$$
0=y^{4}-\left(x^{2} \pm 1\right)^{2}=\left(y^{0}-\left(x^{2} \pm 1\right)\right)\left(y^{2}+\left(x^{2} \pm 1\right)\right) ;
$$

singular models of the Riemann sphere.

ii) $p_{2}^{-1}(0)=\{2, \pm 6\}$, these critical points are the orbit of the fixed points of the order 2 Möbius transformations in $Q_{2}$, therefore each one has ramification order 2 and they correspond to the compact Riemann surface for which its surface group lies inside the quadrangle group of case iv) in Lemma 13. It is the well known quartic Fermat curve:

$$
y^{4}=x^{4}+1
$$

whose automorphism group is isomorphic to $S_{3} \ltimes\left(\mathrm{C}_{4} \times \mathrm{C}_{4}\right)$.

iii) There are two more ramified points on the fiber of the third critical value, $\pm 7 \sqrt{-5}$, each one has ramification order equals 3 (they are zeros of order 2 of the derivative of $p_{2}$ ) and, according to [10], its associated compact Riemann surface admits the following algebraic equation:

$$
y^{4}=x^{3}-1
$$

Its surface group lies inside the quadrangle group of case iii) in Lemma 13 and its automorphism group is an extended group of $G_{2}$ by $\mathrm{C}_{3}$.

In order to obtain the parameter $t$ corresponding to the complete list of algebraic curves belonging to the family $\mathcal{F}_{2}$ for which their surface groups lie in the quadrangle groups $\Gamma_{i}$ inside the triangle groups listed in Lemma 13 or, equivalently, the ones which admit a uniform Belyi function, we will proceed as in the previous section and with the same notation. In this case, we get the values of $t$ we are interested in as the solution of the equation $\frac{t+2}{t-2}=\lambda\left(R_{1}^{i}, R_{2}^{i}, R_{3}^{i}, R_{4}^{i}\right)$, equation obtained imposing that

$$
\lambda\left(R_{1}^{i}, R_{2}^{i}, R_{3}^{i}, R_{4}^{i}\right)=\lambda\left(Q_{1}, Q_{2}, Q_{3}, Q_{4}\right)=\lambda(-2, t, 2, \infty)=\frac{t+2}{t-2}
$$

namely, following the order of the cases of triangle inclusions listed in Lemma 13 and with the notation previously fixed

Proposition 17. A Riemann surface $S_{t} \in \mathcal{F}_{2}$ has the normaliser of its surface group contained in a triangle group or, in other words, $S_{t} \in \mathcal{F}_{2}$ has a uniform Belyi function which factorizes through the quotient by the 
automorphism group $G_{2}$ if and only if $t$ is obtained from the data of one of the following cases:

i) $h_{1}(z)=z^{4}(1-z)$;

$$
\begin{aligned}
& \left(\nu_{1}^{11}, \nu_{2}^{11}, \nu_{3}^{11}, \nu_{4}^{11} ; \nu_{1}^{21}, \nu_{2}^{21} ; \nu_{1}^{31}\right)=(2,1,1,1 ; 4,1 ; 5) . \\
& R_{1}^{1}=\frac{-(135+60 \sqrt{6})^{1 / 3}}{15}+\frac{1}{(135+60 \sqrt{6})^{1 / 3}}-\frac{1}{5}, R_{4}^{1}=1 \text {, } \\
& R_{2}^{1}=\frac{(135+60 \sqrt{6})^{1 / 3}}{30}-\frac{1}{2(135+60 \sqrt{6})^{1 / 3}}-\frac{1}{5}+\frac{\sqrt{-3}}{10}\left(\frac{-(135+60 \sqrt{6})^{1 / 3}}{3}-\frac{5}{(135+60 \sqrt{6})^{1 / 3}}\right), \\
& R_{3}^{1}=\frac{(135+60 \sqrt{6})^{1 / 3}}{30}-\frac{1}{2(135+60 \sqrt{6})^{1 / 3}}-\frac{1}{5}-\frac{\sqrt{-3}}{10}\left(\frac{-(135+60 \sqrt{6})^{1 / 3}}{3}-\frac{5}{(135+60 \sqrt{6})^{1 / 3}}\right) . \\
& \frac{t+2}{t-2}=\lambda\left(R_{1}^{1}, R_{2}^{1}, R_{3}^{1}, R_{4}^{1}\right)=-\frac{1}{5}-\frac{2(135+60 \sqrt{6})^{1 / 3} \sqrt{6}}{75}+\frac{3(135+60 \sqrt{6})^{1 / 3}}{50}-\frac{135+60 \sqrt{6})^{2 / 3}}{150} \\
& +\frac{113 \sqrt{-2}}{480}-\frac{49(135+60 \sqrt{6})^{1 / 3} \sqrt{-3}}{900}+\frac{49(135+60 \sqrt{6})^{1 / 3} \sqrt{-2}}{800}-\frac{49(135+60 \sqrt{6})^{2 / 3} \sqrt{-2}}{7200}
\end{aligned}
$$

therefore

$$
\begin{aligned}
t & =\frac{-164719(135+60 \sqrt{6})^{2 / 3} \sqrt{6}}{8452908}+\frac{299825(135+60 \sqrt{6})^{1 / 3} \sqrt{6}}{19723452}+\frac{766685(135+60 \sqrt{6})^{1 / 3}}{19723452}+ \\
& +\frac{939731(135+60 \sqrt{6})^{2 / 3}}{19723452}-\frac{3631741}{3287242}+\frac{592681(135+60 \sqrt{6})^{2 / 3} \sqrt{-2}}{59170356}-\frac{1329205(135+60 \sqrt{6})^{1 / 3} \sqrt{-2}}{6574484} \\
& +\frac{9712985(135+60 \sqrt{6})^{1 / 3} \sqrt{-3}}{59170356}+\frac{61377(135+60 \sqrt{6})^{2 / 3} \sqrt{-3}}{6574484}-\frac{7452875 \sqrt{-2}}{9861726}
\end{aligned}
$$

ii) $h_{2}(z)=z^{2}(z-1) ; \quad\left(\nu_{1}^{12}, \nu_{2}^{12} ; \nu_{1}^{22}, \nu_{2}^{22} ; \nu_{1}^{32}\right)=(2,1 ; 2,1 ; 3)$.

$R_{1}^{2}=\frac{-1}{3}, R_{2}^{2}=0, R_{3}^{2}=\infty$ and $R_{4}^{2}=1$.

$\lambda\left(R_{1}^{2}, R_{2}^{2}, R_{3}^{2}, R_{4}^{2}\right)=\frac{1}{4}=\frac{t+2}{t-2}$ therefore $t=\frac{10}{3}$.

iii) $h_{3}(z)=z^{3} ; \quad\left(\nu_{1}^{13}, \nu_{2}^{13}, \nu_{3}^{13} ; \nu_{1}^{23} ; \nu_{1}^{33}\right)=(1,1,1 ; 3 ; 3)$.

$R_{1}^{3}=1, R_{2}^{3}=\frac{-1+\sqrt{-3}}{2}, R_{3}^{3}=\frac{-1-\sqrt{-3}}{2}$ and $R_{4}^{3}=\infty$.

$\lambda\left(R_{1}^{3}, R_{2}^{3}, R_{3}^{3}, R_{4}^{3}\right)=\frac{1-\sqrt{-3}}{2}=\frac{t+2}{t-2}$ therefore $t=2 \sqrt{-3}$.

iv) $h_{4}(z)=z^{2} ; \quad\left(\nu_{1}^{14}, \nu_{2}^{14} ; \nu_{1}^{24} ; \nu_{1}^{34}\right)=(1,1 ; 2 ; 2)$.

$R_{1}^{4}=0, R_{2}^{4}=1, R_{3}^{4}=-1, R_{4}^{4}=\infty$.

$\lambda\left(R_{1}^{4}, R_{2}^{4}, R_{3}^{4}, R_{4}^{4}\right)=\frac{1}{2}=\frac{t+2}{t-2}$ therefore $t=-6$.

In this section as in the previous one, for every genus 3 curve $S_{t}$ belonging to the family $\mathcal{F}_{2}$, we work out the 6-holomorphic differential which is the Wronskian $\left(\mathrm{Wr}_{2}\left(S_{t}\right)\right)$ of the basis for the vector space of holomorphic differentials given by $x, y$ and $z$. In order to make the direct computation which is the Wronskian for this basis, we use the meromorphic function $y / z$ as a local parameter at every point of the curve, unless a finite number of them, in the affine neighborhood $z=1$ and, finally, we return it to a global expression what gives us that 
Theorem 18. For every compact Riemann surface $S_{t}$ belonging to the family $\mathcal{F}_{2}$, the Wronskian of the basis $\{x, y, z\}$ of the space of holomorphic differentials is

$$
\mathrm{Wr}_{2}\left(S_{t}\right)=3 x^{2} y^{2} z^{2} t^{2}+2 y^{6} t-12 x^{2} y^{2} z^{2} \text {. }
$$

\section{Case (III). Moduli of the family $\mathcal{F}_{3}$}

The 1-complex dimensional family of genus 3 compact Riemann surfaces admitting an automorphism group $G_{3}$ isomorphic to $\mathrm{D}_{4} \times \mathrm{C}_{2}$ with branching data $(2,2,2,4), \mathcal{F}_{3}$, was already obtained in [10]. Each member of the family is a hyperelliptic curve with algebraic equation given by

$$
S_{t} \equiv\left\{y^{2}=x^{8}-t x^{4}+1=\left(x^{4}-e^{4}\right)\left(x^{4}-1 / e^{4}\right)\right\},
$$

for every $t \in \mathbb{C}-\{ \pm 2\}$ and $t=e^{4}+1 / e^{4}$.

The action of the automorphism group on any surface $S_{t} \in \mathcal{F}_{3}$ can be described as follows:

$$
\begin{aligned}
& a(x, y)=(i x, y), \quad b(x, y)=\left(1 / x, y / x^{4}\right) \\
& \text { and } c(x, y)=(x,-y) \text { with } i=\sqrt{-1}
\end{aligned}
$$

$\mathrm{D}_{4} \times \mathrm{C}_{2} \cong\left\langle\left\{a, b, c: a^{4}=b^{2}=c^{3}=1, b a=a^{3} b, a c=c a\right.\right.$ and $\left.\left.b c=c b\right\}\right\rangle$.

Although this family is composed of hyperelliptic surfaces, therefore they have not a canonical model, we still will use the character table of $\mathrm{D}_{4} \times \mathrm{C}_{2}$ in order to get the number of different surface kernel epimorphisms $\varphi$ from $\Gamma=\left\langle x, y, z \mid x^{2}=y^{2}=z^{2}=(x y z)^{-4}=1\right\rangle$ Fuchsian quadrangle group of signature $[2,2,2,4]$ to the automorphism group $G_{3}$ as we did in the previous sections:

$$
\begin{array}{ccccccccccc}
C & 1 A & 2 A_{1} & 2 B_{1} & 2 B_{6} & 2 A_{2} & 2 A_{3} & 2 B_{3} & 2 B_{4} & 4 D_{1} & 4 D_{2} \\
& i d & a^{2} & b & a b & c & a^{2} c & b c & a b c & a & a c \\
|C| & 1 & 1 & 2 & 2 & 1 & 1 & 2 & 2 & 2 & 2 \\
\psi_{1} & 1 & 1 & 6 & 4 & 1 & 1 & 1 & 1 & 1 & 1 \\
\psi_{2} & 1 & 1 & 1 & 1 & -1 & -1 & -1 & -1 & 1 & -1 \\
\psi_{3} & 1 & 1 & -1 & -1 & 1 & 1 & -1 & -1 & 1 & 1 \\
\psi_{4} & 1 & 1 & -1 & -1 & -1 & -1 & 9 & 1 & 1 & -1 \\
\psi_{5} & 1 & 1 & 1 & -1 & 1 & 1 & 1 & -1 & -1 & -6 \\
\psi_{4} & 1 & 1 & 1 & -1 & -1 & -1 & -1 & 1 & -1 & 1 \\
\psi_{7} & 2 & 1 & -1 & 1 & 1 & 1 & -1 & 1 & -1 & -1 \\
\psi_{8} & 1 & 1 & -1 & 1 & -1 & -1 & 1 & -1 & -1 & 1 \\
\psi_{9} & 2 & -2 & 0 & 0 & 2 & -2 & 0 & 0 & 0 & 0 \\
\psi_{10} & 2 & -2 & 0 & 0 & -2 & 2 & 0 & 0 & 0 & 0 .
\end{array}
$$


We need as well some more information about this group as it is some knowledge about its characteristic subgroups, that is, its commutator subgroup $G_{3}^{\prime}=\left\langle a^{2}\right\rangle \cong \mathrm{C}_{2}$, its center $Z\left(G_{3}\right)=\left\langle a^{2}, c\right\rangle \cong \mathrm{C}_{2} \times \mathrm{C}_{2}$ and $\langle a, c\rangle$ its unique subgroup isomorphic to $\mathrm{C}_{4} \times \mathrm{C}_{2}$. Therefore, any epimorphism $\varphi$ has to apply one of the three order two generators of $\Gamma$ to a central involution and all of them to elements belonging to different conjugacy classes because otherwise, composing $\varphi$ with the quotient epimorphism $\pi: G_{3} \rightarrow G_{3} / Z\left(G_{3}\right)$, one gets a contradiction with the fact that we must have $\pi \circ \varphi\left(x_{1} x_{2} x_{3} x_{4}\right)=1$. By other hand, this central involution can not be $a^{2}$ because $\varphi$ would induce the following epimorphism between the corresponding quotient subgroups

$$
\pi^{\prime}:\left\langle\bar{x}, \bar{y}, \bar{z} \mid \bar{x}^{2}=\bar{y}^{2}=\bar{z}^{4}=\overline{x y z}=1\right\rangle \cong \mathrm{D}_{4} \rightarrow G_{3} / G_{3}^{\prime} \cong \mathrm{C}_{2} \times \mathrm{C}_{2} \times \mathrm{C}_{2}
$$

which is clearly impossible. Thus the number of different epimorphisms equals the number of solutions of the equations $\bar{x}_{1}^{2}=\bar{x}_{2}^{2}=\bar{x}_{3}^{2}=\bar{x}_{4}^{4}=$ $\bar{x}_{1} \bar{x}_{2} \bar{x}_{3} \bar{x}_{4}=1$, where $\bar{x}_{1}, \bar{x}_{2}, \bar{x}_{3}$ belong to any permutation of the conjugacy classes $2 A_{k}, 2 B_{r}$ and $2 B_{s}$ with $k=2,3$ and $r \neq s$; and $\bar{x}_{4}$ to $4 D_{j}(j=1$ and 2). This number $n_{\varphi}$ can be easily calculated by using the well known formula in [8],

$$
n_{\varphi}:=\sum_{j=1}^{2} \sum_{k=2}^{3} \sum_{r \neq s} 6 \frac{\left|2 A_{k}\right||| 2 B_{r}|| 2 B_{s}|| 4 D_{j} \mid}{\left|G_{3}\right|} \sum_{i=1}^{10} \frac{\psi_{i}\left(\bar{x}_{1}\right) \psi_{i}\left(\bar{x}_{2}\right) \psi_{i}\left(\bar{x}_{3}\right) \psi_{i}\left(\bar{x}_{4}\right)}{\psi_{i}(1)^{2}}=192 .
$$

The automorphism group of $\mathrm{D}_{4} \times \mathrm{C}_{2}$ has order $64=8 \times 8$ because the order of the automorphism of the characteristic subgroup $\langle a, c\rangle \cong \mathrm{C}_{4} \times \mathrm{C}_{2}$ is 8 and, independently, the image under any automorphism of $\mathrm{D}_{4} \times \mathrm{C}_{2}$ of $b$ could be any of the 8 non-central involutions. Therefore we obtain the total number of different kernels inside a quadrangle group of signature $[2,2,2,4]$ to be $n_{\varphi} / 64=3$. It is obvious that the epimorphisms can be describe as follows:

Lemma 19. For every Fuchsian group $\Gamma$ with signature $[2,2,2,4]$, there exist three non-equivalent surface kernel epimorphisms $\varphi_{i}: \Gamma \rightarrow \mathrm{D}_{4} \times \mathrm{C}_{2}$ given by

$$
\begin{array}{lll}
\varphi_{1}(x)=c & \varphi_{2}(x)=b & \varphi_{3}(x)=b a c \\
\varphi_{1}(y)=b & \varphi_{2}(y)=b a c & \varphi_{3}(y)=c \\
\varphi_{1}(z)=b a c & \varphi_{2}(z)=c & \varphi_{3}(z)=b .
\end{array}
$$

Generically these kernels belong to biholomorphically inequivalent Riemann surfaces. However it might occur that some of these kernels are conjugate inside $P S L_{2}(\mathbb{R})$, that is, in some bigger Fuchsian group $\Delta$ containing the group $[2,2,2,4]$. It was proved in Lemma 13 that $\Delta$ has to be a triangle group; moreover 
Lemma 20. a) The possible inclusions of a Fuchsian group $\Gamma_{i}$ of signature $[2,2,2,4]$ inside a triangle group $\Delta_{i}$ are the following:
i) $\Gamma_{1}=[2,2,2,4]_{1}$
ii) $\Gamma_{2}=[2,2,2,4]_{2}$
$\leq[2,4,5]=\Delta_{1}$ with index 5 .
iii) $\Gamma_{3}=[2,2,2,4]_{3}$
$\leq[2,4,6]=\Delta_{2}$ with index 3 .
iv) $\Gamma_{4}=[2,2,2,4]_{4}$
with indexes 2 and 3 , respectively.
$\unlhd[2,3,12]=\Delta_{3}$ with index 3 .
$\unlhd[2,4,8]=\Delta_{4} \leq[2,3,8]$

b) In case iii) the three different kernels belong to only one biholomorphically inequivalent Riemann surface, i.e. the orbit of a kernel inside [2,3,12] under conjugation has length 1 . In case iv) we find 2 different Riemann surfaces. In case i) and ii) there are 3 biholomorphically inequivalent Riemann surfaces.

Proof. a) This part was already proved in Lemma 13.

b) As we did in Lemma 4, from the permutation representations in the proof of Lemma 13, we deduce (see [14]) not only the inclusions but the generators of each quadrangle group in terms of the generators of the corresponding triangle group which contains it; for instance, sets of left-coset representatives in these cases are given by:

$$
\begin{gathered}
\left\{\Gamma_{1}, z_{1} \Gamma_{1}, z_{1}^{2} \Gamma_{1}, z_{1}^{3} \Gamma_{1}, z_{1}^{4} \Gamma_{1}, z_{1}^{5} \Gamma_{1}, z_{1}^{6} \Gamma_{1}\right\} ; \quad\left\{\Gamma_{2}, z_{2} \Gamma_{2}, z_{2}^{2} \Gamma_{2}, z_{2}^{3} \Gamma_{2}\right\} ; \\
\left\{\Gamma_{3}, y_{3} \Gamma_{3}, y_{3}^{2} \Gamma_{3}\right\} \quad \text { and } \quad\left\{\Gamma_{4}, y_{4} \Gamma_{4}\right\}
\end{gathered}
$$

where, of course, the notation we are considering is $\Delta_{i}=\left\langle x_{i}, y_{i}, z_{i}\right| x_{i}^{r_{i}}=$ $\left.y_{i}^{l_{i}}=z_{i}^{m_{i}}=x_{i} y_{i} z_{i}=1\right\rangle$. Therefore,

$$
\Gamma_{4}=\left\langle x_{4}, y_{4}^{-1} x_{4} y_{4}, y_{4}^{2} \mid x_{4}^{2}=\left(y_{4}^{-1} x_{4} y_{4}\right)^{2}=\left(y_{4}^{2}\right)^{2}=\left(x_{4} z_{4}^{-2} x_{4}^{-1}\right)^{4}=1\right\rangle
$$

and

$$
\begin{aligned}
\Gamma_{3}=\left\langle x_{3},\right. & y_{3}^{-1} x_{3} y_{3}, y_{3}^{-2} x_{3} y_{3}^{2} \mid \\
& \left.x_{3}^{2}=\left(y_{3}^{-1} x_{3} y_{3}\right)^{2}=\left(y_{3}^{-2} x_{3} y_{3}^{2}\right)^{2}=\left(x_{3} z_{3}^{-3} x_{3}^{-1}\right)^{4}=1\right\rangle .
\end{aligned}
$$

Let us now consider the automorphisms of $\Gamma_{3}$ and $\Gamma_{4}$ given by conjugation by $y_{3} \in \Delta_{3}$ and $y_{4} \in \Delta_{4}$, respectively; that is, $\eta_{j}(\gamma)=y_{j}^{-1} \gamma y_{j}$ for any $\gamma \in \Gamma_{j}$ $(j=3$ and 4$)$. Then, we have that there exists an order 2 automorphism $\tau$ of $G_{3} \cong \mathrm{D}_{4} \times \mathrm{C}_{2}$ such that

$$
\begin{aligned}
& \varphi_{1} \circ \eta_{4}\left(x_{4}\right)=\varphi_{1}\left(y_{4}^{-1} x_{4} y_{4}\right)=b=\tau(b a c)=\tau \circ \varphi_{3}\left(x_{4}\right) \\
& \varphi_{1} \circ \eta_{4}\left(y_{4}^{-1} x_{4} y_{4}\right)=\varphi_{1}\left(y_{4}^{-2} x_{4} y_{4}^{2}\right)=(b a c)^{-1} c(b a c)=c=\tau(c)=\tau \circ \varphi_{3}\left(y_{4}^{-1} x_{4} y_{4}\right) \\
& \varphi_{1} \circ \eta_{4}\left(y_{4}^{2}\right)=\varphi_{1}\left(y_{4}^{2}\right)=b a c=\tau(b)=\tau \circ \varphi_{3}\left(y_{4}^{2}\right) \\
& \varphi_{2} \circ \eta_{4}\left(x_{4}\right)=\varphi_{2}\left(y_{4}^{-1} x_{4} y_{4}\right)=b a c=\tau(b)=\tau \circ \varphi_{2}\left(x_{4}\right) \\
& \varphi_{2} \circ \eta_{4}\left(y_{4}^{-1} x_{4} y_{4}\right)=\varphi_{2}\left(y_{4}^{-2} x_{4} y_{4}^{2}\right)=c^{-1} b c=b=\tau(b a c)=\tau \circ \varphi_{2}\left(y_{4}^{-1} x_{4} y_{4}\right) \\
& \varphi_{2} \circ \eta_{4}\left(y_{4}^{2}\right)=\varphi_{2}\left(y_{4}^{2}\right)=c=\tau(c)=\tau \circ \varphi_{2}\left(y_{4}^{2}\right) .
\end{aligned}
$$


Therefore,

$$
y_{4}^{-1}\left(\operatorname{Ker}\left(\varphi_{3}\right)\right) y_{4}=\operatorname{Ker}\left(\varphi_{1}\right) \text { and } y_{4}^{-1}\left(\operatorname{Ker}\left(\varphi_{2}\right) y_{4}=\operatorname{Ker}\left(\varphi_{2}\right) .\right.
$$

On the other hand,

$$
\begin{aligned}
& \varphi_{1} \circ \eta_{3}\left(x_{3}\right)=\varphi_{1}\left(y_{3}^{-1} x_{3} y_{3}\right)=b=\varphi_{2}\left(x_{3}\right) \\
& \varphi_{1} \circ \eta_{3}\left(y_{3}^{-1} x_{3} y_{3}\right)=\varphi_{1}\left(y_{3}^{-2} x_{3} y_{3}^{2}\right)=b a c=\varphi_{2}\left(y_{3}^{-1} x_{3} y_{3}\right) \\
& \varphi_{1} \circ \eta_{3}\left(y_{3}^{-2} x_{3} y_{3}^{2}\right)=\varphi_{1}\left(x_{3}\right)=c=\varphi_{2}\left(y_{3}^{-2} x_{3} y_{3}^{2}\right)
\end{aligned}
$$

and so on. Then, we have

$$
y_{3}^{-1}\left(\operatorname{Ker}\left(\varphi_{2}\right)\right) y_{3}=\operatorname{Ker}\left(\varphi_{1}\right) \quad \text { and } \quad y_{3}^{-1}\left(\operatorname{Ker}\left(\varphi_{3}\right)\right) y_{3}=\operatorname{Ker}\left(\varphi_{2}\right) \text {. }
$$

Proposition 21. $S_{t}$ and $S_{t^{\prime}}$ belonging to $\mathcal{F}_{3}$ are isomorphic if and only if $p_{3}\left(t^{\prime}\right)=p_{3}(t)$, where $p_{3}: \mathbb{C P}^{1} \rightarrow \mathbb{C P}^{1}$ is given by $p_{3}(z)=z^{2}$.

Proof. The proof follows from two steps:

i) According to Lemma 13 there is no two subgroups with signature $[2,2,2,4]$ included and non-conjugate in $N(K)$ being $K$ the surface group of $S_{t}$, therefore $S_{t}$ and $S_{t^{\prime}}$ in $\mathcal{F}_{3}$ are isomorphic only if $S_{t} / G_{3}$ is isomorphic to $S_{t^{\prime}} / G_{3}$.

ii) Now, the proof of the proposition follows from i) together with Remark 2 and Lemma 1, because in this section we have the hyperelliptic involution $c$ that is a central element of the automorphism group of $S_{t} \in \mathcal{F}_{3}$, $\mathrm{D}_{4} \times\langle c\rangle$, hence the subgroup $\mathrm{D}_{4}$ projects to automorphisms of the quotient surface $S_{t} /\langle c\rangle, \widetilde{\mathrm{D}}_{4}$. Moreover, as $\langle\widetilde{a}\rangle$ is a normal subgroup of $\widetilde{\mathrm{D}}_{4}$, $<\widetilde{b}>$ projects again to automorphisms of the quotient surface $S_{t} /<b, c>$. Summarizing, the invariant 16 degree meromorphic function $f_{3}$ on $S_{t} \in \mathcal{F}_{3}$ can be obtained as the composition of the 3 quotient morphisms mentioned above; more explicitly,

$$
f_{3}(x, y)=x^{4}+\frac{1}{x^{4}} .
$$

By other hand, now using (5.1) we can get a representative for each of the 4 orbits of fixed points and their images under $f_{3}$ on the Riemann sphere, which are

$$
\begin{aligned}
P_{1}:=\left(\sqrt{-i}, \frac{1+e^{4}}{e^{2}}\right) & \longmapsto Q_{1}=-2 \\
P_{2}:=(e, 0) & \longmapsto Q_{2}=e^{4}+1 / e^{4}=t \\
P_{3}:=\left(1, \frac{\left(1-e^{4}\right) i}{e^{2}}\right) & \longmapsto Q_{3}=2 \\
P_{4}:=(0,1) & \longmapsto Q_{4}=\infty,
\end{aligned}
$$

where $P_{4}$ is one of the 4 fixed points of $a, P_{3}$ is one of the 4 fixed points of $b$, $P_{2}$ is one of the 8 fixed points of $c$ and, finally, $P_{1}$ is one of the 4 fixed points of $b a c$. 
If we consider the order 3 Möbius transformation permuting the three critical values with ramification order equals 2 , we obtain the three nonequivalent epimorphisms that as was proved in Lemma 19 are not equivalent or, in other words, they do not differ by post-composition with an automorphism of $G_{3}$ because neither $b$ nor $b a c$ are central elements as it is $c$. Therefore, they do not lift to isomorphisms of $S_{t}$. On the other hand, if we consider the order 2 Möbius transformation that interchange the two critical values 2 and -2 , there exists $\tau \in$ Aut $\left(G_{3}\right)$ such that $\tau(b)=b a c$ or, in other words, $\tau$ makes commutative the group diagram in Lemma 1 corresponding to this Möbius transformation and these normal coverings, so it lifts to an isomorphism between $S_{t}$ and $S_{-t}$. The last statement follows from the fact that the cross ratio is $\lambda\left(Q_{1}, Q_{2}, Q_{3}, Q_{4}\right)=\frac{t+2}{t-2}=\lambda_{3}$ which implies that $t=\frac{2\left(\lambda_{3}+1\right)}{\lambda_{3}-1}=g\left(\lambda_{3}\right), \lambda\left(Q_{3}, Q_{2}, Q_{1}, Q_{4}\right)=1 / \lambda_{3}$ and $g\left(1 / \lambda_{3}\right)=-t$ which finishes the proof. More explicitly, an isomorphism between $S_{t}$ and $S_{-t}$ belonging to $\mathcal{F}_{3}$ is given by $\epsilon(x, y)=(\sqrt{i} x, y)$ because if $(x, y) \in S_{t}$ we have $y^{2}=(\sqrt{i} x)^{8}-t(\sqrt{i} x)^{4}+1$ or, in other words, $(\sqrt{i} x, y) \in S_{-t}$.

Moreover,

Theorem 22. Let $Q_{3}$ be the order 2 group of Möbius transformations generated by $z \mapsto-z$, and let $\left[S_{t}\right]$ be the isomorphism class of any genus 3 compact Riemann surface belonging to the family $\mathcal{F}_{3}$. Then the mapping

$$
\Phi: t \mapsto\left[S_{t}\right]
$$

is an holomorphic injection between the analytic space $(\mathbb{C}-\{ \pm 2\}) / Q_{3}$ and the moduli space $\mathcal{M}_{3}$ of compact Riemann surfaces of genus 3. Its image is the subvariety of $\mathcal{M}_{3}$ consisting of those surfaces admitting an automorphism group isomorphic to $\mathrm{D}_{4} \times \mathrm{C}_{2}$.

Proof. As in the previous section it follows directly from [11] and Proposition 21.

In order to obtain the parameter $t$ corresponding to the complete list of algebraic curves belonging to the family $\mathcal{F}_{3}$ for which their surface groups lie in the quadrangle groups $\Gamma_{i}$ inside the triangle groups listed in part $a$ ) of Lemma 20 or, equivalently, the ones which admit a uniform Belyi function that factorizes through the quotient by the automorphism group, we will proceed as in the previous sections and with the same notation. In this case, we get the values of $t$ we are interested in as the orbit under $\langle N\rangle$, $N(z)=\frac{2(z+6)}{2-z}$, of the solution of the equation $\frac{t+2}{t-2}=\lambda\left(R_{1}^{i}, R_{2}^{i}, R_{3}^{i}, R_{4}^{i}\right)$ or, equivalently, solving $\frac{4}{t+2}=\lambda\left(R_{1}^{i}, R_{2}^{i}, R_{3}^{i}, R_{4}^{i}\right)$ and $\frac{2-t}{4}=\lambda\left(R_{1}^{i}, R_{2}^{i}, R_{3}^{i}, R_{4}^{i}\right)$ as well; namely, following the order of the cases of triangle inclusions listed in Lemma 20 and with the notation previously fixed, 
Genus 3 Normal COVERINGS of the Riemann sphere Branched over 4 POINTS 443

Proposition 23. A Riemann surface $S_{t} \in \mathcal{F}_{3}$ has the normalizer of its surface group contained in a triangle group or, in other words, $S_{t} \in \mathcal{F}_{3}$ has a uniform Belyi function which factorizes through the quotient by the automorphism group $G_{3}$ if and only if $t$ is obtained from the data of one of the following cases:

i) $h_{1}(z)=z^{4}(1-z) ; \quad\left(\nu_{1}^{11}, \nu_{2}^{11}, \nu_{3}^{11}, \nu_{4}^{11} ; \nu_{1}^{21}, \nu_{2}^{21} ; \nu_{1}^{31}\right)=(2,1,1,1 ; 4,1 ; 5)$.

$R_{1}^{1}=\frac{-(135+60 \sqrt{6})^{1 / 3}}{15}+\frac{1}{(135+60 \sqrt{6})^{1 / 3}}-\frac{1}{5}, \quad R_{4}^{1}=1$,

$R_{2}^{1}=\frac{(135+60 \sqrt{6})^{1 / 3}}{30}-\frac{1}{2(135+60 \sqrt{6})^{1 / 3}}-\frac{1}{5}+\frac{\sqrt{-3}}{10}\left(\frac{-(135+60 \sqrt{6})^{1 / 3}}{3}-\frac{5}{(135+60 \sqrt{6})^{1 / 3}}\right)$,

$R_{3}^{1}=\frac{(135+60 \sqrt{6})^{1 / 3}}{30}-\frac{1}{2(135+60 \sqrt{6})^{1 / 3}}-\frac{1}{5}-\frac{\sqrt{-3}}{10}\left(\frac{-(135+60 \sqrt{6})^{1 / 3}}{3}-\frac{5}{(135+60 \sqrt{6})^{1 / 3}}\right)$.

$\frac{t+2}{t-2}=\lambda\left(R_{1}^{1}, R_{2}^{1}, R_{3}^{1}, R_{4}^{1}\right)=-\frac{1}{5}-\frac{2(135+60 \sqrt{6})^{1 / 3} \sqrt{6}}{75}+\frac{3(135+60 \sqrt{6})^{1 / 3}}{50}-\frac{135+60 \sqrt{6})^{2 / 3}}{150}$ $+\frac{113 \sqrt{-2}}{480}-\frac{49(135+60 \sqrt{6})^{1 / 3} \sqrt{-3}}{900}+\frac{49(135+60 \sqrt{6})^{1 / 3} \sqrt{-2}}{800}-\frac{49(135+60 \sqrt{6})^{2 / 3} \sqrt{-2}}{7200}$

therefore

$$
\begin{aligned}
t= & \frac{-164719(135+60 \sqrt{6})^{2 / 3} \sqrt{6}}{8452908}+\frac{299825(135+60 \sqrt{6})^{1 / 3} \sqrt{6}}{19723452}+\frac{766685(135+60 \sqrt{6})^{1 / 3}}{19723452}+ \\
+ & \frac{939731(135+60 \sqrt{6})^{2 / 3}}{19723452}-\frac{3631741}{3287242}+\frac{592681(135+60 \sqrt{6})^{2 / 3} \sqrt{-2}}{59170356}-\frac{7452875 \sqrt{-2}}{9861726} \\
- & \frac{1329205(135+60 \sqrt{6})^{1 / 3} \sqrt{-2}}{6574484}+\frac{9712985(135+60 \sqrt{6})^{1 / 3} \sqrt{-3}}{59170356}+\frac{61377(135+60 \sqrt{6})^{2 / 3} \sqrt{-3}}{6574484} \\
N(t)= & \frac{1249(135+60 \sqrt{6})^{2 / 3} \sqrt{6}}{18432}-\frac{2525(135+60 \sqrt{6})^{1 / 3} \sqrt{6}}{6144}+\frac{515(135+60 \sqrt{6})^{1 / 3}}{768}-\frac{203}{64}- \\
& -\frac{49(135+60 \sqrt{6})^{2 / 3}}{192}+\frac{49(135+60 \sqrt{6})^{2 / 3} \sqrt{-3}}{192}+\frac{565(135+60 \sqrt{6})^{1 / 3} \sqrt{-3}}{2304}+ \\
& +\frac{415(135+60 \sqrt{6})^{1 / 3} \sqrt{-2}}{2048}+\frac{5857(135+60 \sqrt{6})^{2 / 3} \sqrt{-2}}{18432}-\frac{6875 \sqrt{-2}}{3072} \\
N^{2}(t)= & \frac{14}{5}+\frac{8(135+60 \sqrt{6})^{1 / 3} \sqrt{6}}{75}-\frac{6(135+60 \sqrt{6})^{1 / 3}}{25}+\frac{2(135+60 \sqrt{6})^{2 / 3}}{75}+ \\
& +\frac{49(135+60 \sqrt{6})^{1 / 3} \sqrt{-3}}{225}-\frac{49(135+60 \sqrt{6})^{2 / 3} \sqrt{-2}}{200}-\frac{113 \sqrt{-2}}{120}+\frac{49(135+60 \sqrt{6})^{2 / 3} \sqrt{-2}}{1800} .
\end{aligned}
$$

ii) $h_{2}(z)=z^{2}(z-1) ; \quad\left(\nu_{1}^{12}, \nu_{2}^{12} ; \nu_{1}^{22}, \nu_{2}^{22} ; \nu_{1}^{32}\right)=(2,1 ; 2,1 ; 3)$.

$R_{1}^{2}=\frac{-1}{3}, R_{2}^{2}=0, R_{3}^{2}=\infty$ and $R_{4}^{2}=1$.

$\lambda\left(R_{1}^{2}, R_{2}^{2}, R_{3}^{2}, R_{4}^{2}\right)=\frac{1}{4}=\frac{t+2}{t-2}$ therefore $t=\frac{10}{3}, N(t)=-14, N^{2}(t)=-1$.

iii) $h_{3}(z)=z^{3} ; \quad\left(\nu_{1}^{13}, \nu_{2}^{13}, \nu_{3}^{13} ; \nu_{1}^{23} ; \nu_{1}^{33}\right)=(1,1,1 ; 3 ; 3)$.

$R_{1}^{3}=1, R_{2}^{3}=\frac{-1+\sqrt{-3}}{2}, R_{3}^{3}=\frac{-1-\sqrt{-3}}{2}$ and $R_{4}^{3}=\infty$.

$\lambda\left(R_{1}^{3}, R_{2}^{3}, R_{3}^{3}, R_{4}^{3}\right)=\frac{1-\sqrt{-3}}{2}=\frac{t+2}{t-2}$

therefore $t=2 \sqrt{-3}, N(t)=N^{2}(t)=2 \sqrt{-3}$.

iv) $h_{4}(z)=z^{2} ; \quad\left(\nu_{1}^{13}, \nu_{2}^{14} ; \nu_{1}^{24} ; \nu_{1}^{34}\right)=(1,1 ; 2 ; 2)$.

$R_{1}^{4}=0, R_{2}^{4}=1, R_{3}^{4}=-1 \quad$ and $R_{4}^{4}=\infty$.

$\lambda\left(R_{1}^{4}, R_{2}^{4}, R_{3}^{4}, R_{4}^{4}\right)=\frac{1}{2}=\frac{t+2}{t-2}$ therefore $t=-6 ; N(z)=0, N^{2}(z)=-6$. 


\section{Case (IV). Moduli of the family $\mathcal{F}_{4}$}

The 1-complex dimensional family of genus 3 compact Riemann surfaces admitting an automorphism group $G_{4}$ isomorphic to $\mathrm{D}_{6}$ with branching data $(2,2,2,6), \mathcal{F}_{4}$, was already obtained in [10]. Each member of this family is a hyperelliptic curve with algebraic equation given by

$$
S_{t} \equiv\left\{y^{2}=x^{7}-t x^{4}+x=x\left(x^{3}-e^{3}\right)\left(x^{3}-\frac{1}{e^{3}}\right)\right\}
$$

for every $t \in \mathbb{C}-\{ \pm 2\}$ and $t=e^{3}+1 / e^{3}$.

The action of the automorphism group on any surface $S_{t} \in \mathcal{F}_{4}$ can be described as follows:

$$
a(x, y)=\left(\zeta_{3} x,-y\right) \text { and } b(x, y)=\left(\frac{1}{x}, \frac{y}{x^{4}}\right) \text { with } \zeta_{3}=\exp (2 \pi \sqrt{-1} / 3) .
$$

Let us observe that $a^{3}(x, y)=(x,-y)$ is the hyperelliptic involution and

$$
\mathrm{D}_{6} \cong\left\langle\left\{a, b: a^{6}=b^{2}=1 \text {, and } b a=a^{5} b\right\}\right\rangle .
$$

Although this family is composed of hyperelliptic surfaces as in the previous section, therefore they have not either a canonical model, we still will use the character table of $\mathrm{D}_{6}$ in order to get the number of different surface kernel epimorphisms $\varphi$ from every $\Gamma=\left\langle x, y, z \mid x^{2}=y^{2}=z^{2}=(x y z)^{-6}=1\right\rangle$ a Fuchsian quadrangle group of signature $[2,2,2,6]$ to the automorphism group:

$\begin{array}{ccccccc}C & 1 A & 2 A_{1} & 2 A_{2} & 2 C & 3 A & 6 A \\ C \mid & 1 & 3 & 3 & 1 & 2 & 2 \\ & \text { id } & b & b a & c & a^{2} & a \\ \psi_{1} & 1 & 1 & 1 & 1 & 1 & 1 \\ \psi_{2} & 1 & -1 & -1 & 1 & 1 & 1 \\ \psi_{3} & 1 & 1 & -1 & -1 & 1 & -1 \\ \psi_{4} & 1 & -1 & 1 & -1 & 1 & -1 \\ \psi_{5} & 2 & 0 & 0 & -2 & -1 & 1 \\ \psi_{6} & 2 & 0 & 0 & 2 & -1 & 1 .\end{array}$

Let us observe, first of all, that any epimorphism $\varphi$ has to apply one of the three order two generators of $\Gamma$ to the central involution $a^{3}$ and all of them to elements belonging to different conjugacy classes because otherwise, composing $\varphi$ with the quotient epimorphism $\pi: G_{4} \rightarrow G_{4} / Z\left(G_{4}\right)$, where $Z\left(G_{4}\right)=<a^{3}>$ denotes the center of $G_{4}$, one gets a contradiction with the fact that we must have $\pi \circ \varphi\left(x_{1} x_{2} x_{3} x_{4}\right)=\pi \circ \varphi(1)=1$. Thus the number of different epimorphisms equals the number of solutions of the equations $\bar{x}_{1}^{2}=\bar{x}_{2}^{2}=\bar{x}_{3}^{2}=\bar{x}_{4}^{6}=\bar{x}_{1} \bar{x}_{2} \bar{x}_{3} \bar{x}_{4}=1$, where $\bar{x}_{1}$ and $\bar{x}_{2}$ belong to the conjugacy classes $2 A_{j}$, for $j=1$ or $2 ; \bar{x}_{3}$ belongs to $2 B$ and $\bar{x}_{4}$ belongs to $6 A$. 
This number $n_{\varphi}$ can be easily calculated by using the very much mentioned formula in [8],

$$
n_{\varphi}:=\sum_{j=1}^{2} \sum_{k=1}^{2} 3 \frac{\left|2 A_{j}\right||| 2 A_{k}|| 2 B|| 6 A \mid}{\left|G_{4}\right|} \sum_{i=1}^{5} \frac{\psi_{i}\left(\bar{x}_{1}\right) \psi_{i}\left(\bar{x}_{2}\right) \psi_{i}\left(\bar{x}_{3}\right) \psi_{i}\left(\bar{x}_{4}\right)}{\psi_{i}(1)^{2}}=36 .
$$

The automorphism group of $\mathrm{D}_{6}$ has order $12=6 \times 2$ because it is obviously isomorphic to $\mathrm{C}_{6} \times \mathrm{C}_{2}$. Therefore we obtain the total number of different kernels inside the $[2,2,2,6]$ quadrangle group to be $n_{\varphi} / 12=3$. It is easy to check that the three non-equivalent epimorphisms can be given in the following way:

Lemma 24. For every Fuchsian group $\Gamma$ with signature $[2,2,2,6]$, there exist three non-equivalent surface kernel epimorphisms $\varphi_{i}: \Gamma \rightarrow \mathrm{D}_{6}$ given by

$$
\begin{array}{lll}
\varphi_{1}(x)=a^{3} & \varphi_{2}(x)=b & \varphi_{3}(x)=b a^{4} \\
\varphi_{1}(y)=b & \varphi_{2}(y)=b a^{4} & \varphi_{3}(y)=a^{3} \\
\varphi_{1}(z)=b a^{4} & \varphi_{2}(z)=a^{3} & \varphi_{3}(z)=b .
\end{array}
$$

As we exposed in the previous sections, generically this kernels belong to biholomorphically inequivalent Riemann surfaces. However it might occur that some of these kernels are conjugate inside $P S L_{2}(\mathbb{R})$, that is, in some bigger Fuchsian group $\Delta$ that contains at least a Fuchsian group with signature $[2,2,2,6]$. With analogous notation and proceeding in a similar way as we did for proving Lemma 20 and, in this case, with permutation representations given by

$$
\begin{aligned}
& \theta_{1}:[2,4,6] \rightarrow S_{4} \\
& x_{1} \mapsto(1)(4)(23) \\
& \theta_{2}:[2,3,18] \rightarrow S_{3} \\
& x_{2} \mapsto(1)(2)(3) \\
& \theta_{3}:[2,4,12] \rightarrow S_{2} \\
& y_{1} \mapsto(1234) \\
& y_{2} \quad \mapsto(123) \\
& x_{3} \mapsto(1)(2) \\
& z_{1} \mapsto(143)(2) \\
& z_{2} \mapsto(132) \\
& y_{3} \mapsto(12) \\
& z_{3} \mapsto(12) \text {, }
\end{aligned}
$$

one obtains:

Lemma 25. a) The possible inclusions of a Fuchsian group $\Gamma_{i}$ of signature $[2,2,2,6]$ inside a triangle group $\Delta_{i}$ are the following

$$
\begin{aligned}
& \text { i) } \Gamma_{1}=[2,2,2,6]_{1} \leq[2,4,6]=\Delta_{1} \text { with index } 4 \text {. } \\
& \text { ii) } \Gamma_{2}=[2,2,2,6]_{2} \unlhd[2,3,18]=\Delta_{2} \text { with index } 3 \text {. } \\
& \text { iii) } \Gamma_{3}=[2,2,2,6]_{3} \unlhd[2,4,12]=\Delta_{3} \text { with index } 2 \text {. }
\end{aligned}
$$

b) In case ii) the three different kernels belong to only one biholomorphically inequivalent Riemann surface, i.e. the orbit of a kernel inside $[2,3,18]$ under conjugation has length 1 . In case iii) we find 2 different Riemann surfaces. In case i) there are 3 biholomorphically inequivalent Riemann surfaces. 
Proposition 26. $S_{t}$ and $S_{t^{\prime}}$ belonging to $\mathcal{F}_{4}$ are isomorphic if and only if $p_{4}\left(t^{\prime}\right)=p_{4}(t)$, where $p_{4}: \mathbb{C P}^{1} \rightarrow \mathbb{C P}^{1}$ is given by $p_{4}(z)=z^{2}$.

Proof. The proof is the same as the corresponding one in the preceding sections with the suitable changes to this case:

i) According to Lemma 25 there are not two subgroups with signature $[2,2,2,6]$ included and non-conjugate in $N(K)$, being $K$ the surface group of $S_{t}$. Therefore $S_{t}$ and $S_{t^{\prime}}$ in $\mathcal{F}_{4}$ are isomorphic only if $S_{t} / G_{4}$ is isomorphic to $S_{t^{\prime}} / G_{4}$.

ii) Now, the proof of the proposition follows again from i) together with Remark 2 and Lemma 1 because as in the previous section the hyperelliptic involution $a^{3}$ is a central element of the automorphism group of $S_{t} \in \mathcal{F}_{4}, \mathrm{D}_{6}$, so the subgroup $\mathrm{D}_{6} /<a^{3}>\cong \mathrm{D}_{3}$ projects to automorphisms of the quotient surface $S_{t} /<a^{3}>$. Then, as $<\widetilde{a}>$ is a normal subgroup of $\widetilde{\mathrm{D}}_{3},<\widetilde{b}>$ projects again to automorphisms of the quotient surface $S_{t} /<a>$. Summarizing, the invariant 12 degree meromorphic function $f_{4}$ on $S_{t} \in \mathcal{F}_{4}$ is the composition of the 3 quotient morphisms mentioned above; more explicitly,

$$
f_{4}(x, y)=x^{3}+\frac{1}{x^{3}}
$$

On the other hand, now using (6.1) we can get a representative for each of the 4 orbits of fixed points and their images under $f_{4}$ on the Riemann sphere, which are

$$
\begin{aligned}
& P_{1}:=\left(-1, \frac{i\left(e^{3}+1\right)}{e^{3 / 2}}\right) \longmapsto Q_{1}=-2 \\
& P_{2}:=(e, 0) \longmapsto Q_{2}=e^{3}+1 / e^{3}=t \\
& P_{3}:=\left(1, \frac{\left(e^{3}-1\right) i}{e^{3 / 2}}\right) \longmapsto Q_{3}=2 \\
& P_{4}:=(0,0) \longmapsto Q_{4}=\infty,
\end{aligned}
$$

where $P_{4}$ is one of the 2 fixed points of $a, P_{3}$ is one of the 4 fixed points of $b, P_{2}$ is one of the 6 fixed points of the hyperelliptic involution $a^{3}$ different from the fixed points of $a$ and, finally, $P_{1}$ is another fixed point of $b$ belonging to a different orbit than $P_{3}$. If we consider the order 3 Möbius transformations permuting the three critical values with ramification order equals 2, we obtain the three non-equivalent epimorphisms that as was proved in Lemma 24 are not equivalent or, in other words, they do not differ by post-composition with an automorphism of $G_{4}$. Therefore, they do not lift to isomorphisms of $S_{t}$. On the other hand, if we consider the order 2 Möbius transformation that interchanges the two critical values 2 and -2 , there exists $\tau \in \operatorname{Aut}\left(G_{4}\right)$ such that $\tau(b)=b a^{2}$ or, in other words, $\tau$ makes 
commutative the group diagram in Lemma 1 corresponding to this Möbius transformation and these normal coverings, so it lifts to an isomorphism between $S_{t}$ and $S_{-t}$. The last statement now follows from the fact that the cross ratio is $\lambda\left(Q_{1}, Q_{2}, Q_{3}, Q_{4}\right)=\frac{t+2}{t-2}=\lambda_{4}$ which implies that $t=\frac{2\left(\lambda_{4}+1\right)}{\lambda_{4}-1}=g\left(\lambda_{4}\right)$, $\lambda\left(Q_{3}, Q_{2}, Q_{1}, Q_{4}\right)=\frac{1}{\lambda_{4}}$ and $g\left(\frac{1}{\lambda_{4}}\right)=-t$ which finishes the proof. Moreover, an isomorphism between $S_{t}$ and $S_{-t}$ belonging to $\mathcal{F}_{4}$ is, obviously, given by

$$
\epsilon(x, y)=(-x, i y)
$$

Consequently, we have

Theorem 27. Let $Q_{4}$ be the order 3 group of Möbius transformations generated by $z \mapsto \frac{2(z+6)}{2-z}$, and let $\left[S_{t}\right]$ be the isomorphism class of any genus 3 compact Riemann surface belonging to the family $\mathcal{F}_{4}$. Then the mapping

$$
\Phi: t \mapsto\left[S_{t}\right]
$$

is a holomorphic injection between the analytic space $(\mathbb{C}-\{ \pm 2\}) / Q_{4}$ and the moduli space $\mathcal{M}_{3}$ of compact Riemann surfaces of genus 3 . Its image is the subvariety of $\mathcal{M}_{3}$ consisting of those surfaces admitting an automorphism group isomorphic to $\mathrm{D}_{6}$.

Proof. As in the previous sections it follows directly from Proposition 26 and [11].

Remark 28. $p_{4}: \mathbb{C P}^{1} \rightarrow \mathbb{C P}^{1}$ is a Belyi function with critical values 0 and $\infty$. Among the corresponding curves to the set of preimages of the critical values, the unique non singular one corresponds to $p_{2}^{-1}(0)=\{0\}$. This algebraic curve has equation in $\mathcal{F}_{4}$ given by

$$
y^{2}=x\left(x^{6}+1\right)
$$

and its automorphism group is generated by

$$
a(x, y)=\left(\mu^{2} x, \mu y\right), \text { with } \mu=\exp (2 \pi \sqrt{-1} / 6), \quad b(x, y)=\left(\frac{1}{x}, \frac{y}{x^{4}}\right)
$$

and the hyperelliptic involution, that is, its automorphism group is isomorphic to $\mathrm{D}_{6} \times \mathrm{C}_{2}$.

In order to obtain the parameter $t$ corresponding to the complete list of algebraic curves belonging to the family $\mathcal{F}_{4}$ for which their surface groups lie in the quadrangle groups $\Gamma_{i}$ inside the triangle groups listed in part $a$ ) of Lemma 25 or, equivalently, the ones which admit a uniform Belyi function that factorizes through the quotient by the automorphism group, we will 
proceed as in the previous sections and with the same notation. We get the values of $t$ we are interested in as the orbit under $N(z)=\frac{2(z+6)}{z-2}$ to the solution of the equation $\frac{t+2}{t-2}=\lambda\left(R_{1}^{i}, R_{2}^{i}, R_{3}^{i}, R_{4}^{i}\right)$, equation obtained imposing that $\lambda\left(R_{1}^{i}, R_{2}^{i}, R_{3}^{i}, R_{4}^{i}\right)=\lambda\left(Q_{1}, Q_{2}, Q_{3}, Q_{4}\right)=\lambda(-2, t, 2, \infty)=\frac{t+2}{t-2} ;$ namely, following the order of the cases of triangle inclusions listed in Lemma 25 and with the previously fixed notation, we have

Proposition 29. A Riemann surface $S_{t} \in \mathcal{F}_{4}$ has the normalizer of its surface group contained in a triangle group or, in other words, $S_{t} \in \mathcal{F}_{4}$ has a uniform Belyi function which factorizes through the quotient by the automorphism group $G_{4}$ if and only if $t$ is obtained from the data of one of the following cases:

i) $h_{1}(z)=z^{3}(1-z) ; \quad\left(\nu_{1}^{11}, \nu_{2}^{11}, \nu_{3}^{11} ; \nu_{1}^{21}, \nu_{2}^{21} ; \nu_{1}^{31}\right)=(2,1,1 ; 3,1 ; 4)$.

$R_{1}^{1}=\frac{-1+\sqrt{-2}}{4}, R_{2}^{1}=0, R_{3}^{1}=\frac{-1-\sqrt{-2}}{4}$ and $R_{4}^{1}=1$.

$\lambda\left(R_{1}^{1}, R_{2}^{1}, R_{3}^{1}, R_{4}^{1}\right)=\frac{17-56 \sqrt{-2}}{81}=\frac{t+2}{t-2}$ therefore $t=\frac{-7 \sqrt{-2}}{4}$;

$N\left(\frac{-7 \sqrt{-2}}{4}\right)=\frac{-94-224 \sqrt{-2}}{81}$ and $N^{2}\left(\frac{-7 \sqrt{-2}}{4}\right)=\frac{94-224 \sqrt{-2}}{81}$.

ii) $h_{2}(z)=z^{3} ; \quad\left(\nu_{1}^{12}, \nu_{2}^{12}, \nu_{3}^{12} ; \nu_{1}^{22} ; \nu_{1}^{32}\right)=(1,1,1 ; 3 ; 3)$.

$R_{1}^{2}=1, R_{2}^{2}=\frac{-1+\sqrt{-3}}{2}, R_{3}^{2}=\frac{-1-\sqrt{-3}}{2}$ and $R_{4}^{2}=\infty$;

$\lambda\left(R_{1}^{2}, R_{2}^{2}, R_{3}^{2}, R_{4}^{2}\right)=\frac{1-\sqrt{-3}}{2}=\frac{t+2}{t-2}$ therefore $t=2 \sqrt{-3}$.

iii) $h_{3}(z)=z^{2} ; \quad\left(\nu_{1}^{13}, \nu_{2}^{13} ; \nu_{1}^{23} ; \nu_{1}^{33}\right)=(1,1 ; 2 ; 2)$.

$R_{1}^{3}=1, R_{2}^{3}=-1, R_{3}^{3}=0$ and $R_{4}^{3}=\infty$.

$\lambda\left(R_{1}^{3}, R_{2}^{3}, R_{3}^{3}, R_{4}^{3}\right)=\frac{1}{2}=\frac{t+2}{t-2}$ therefore $t=-6, N(z)=0$ and $N^{2}(z)=-6$.

\section{Case (V). Moduli of the family $\mathcal{F}_{5}$}

For every $t \in \mathbb{C}-\{0,1\}$, the non singular algebraic curve described in [10] as a member of the family $\mathcal{F}_{5}$ can be written as:

$$
S_{t} \equiv\left\{y^{3}=\left(x^{2}-1\right)\left(x^{2}-e^{2}\right)=x^{4}-(1+t) x^{2}+t\right\} \subseteq \mathbb{C}^{2} .
$$

The action of the automorphism group on any surface $S_{t} \in \mathcal{F}_{5}$ can be described as follows:

$$
\begin{gathered}
a(x, y)=\left(-x, \zeta_{3} y\right) \text { with } \zeta_{3}=\exp (2 \pi \sqrt{-1} / 3) ; \text { and } \\
\mathrm{C}_{6} \cong\left\langle\left\{a: a^{6}=1\right\}\right\rangle .
\end{gathered}
$$

In order to calculate the number of different epimorphisms $\varphi$ from $\Gamma=$ $\left\langle x, y, z \mid x^{2}=y^{3}=z^{3}=(x y z)^{-6}=1\right\rangle$ Fuchsian quadrangle group $[2,3,3,6]$ 
to the automorphism group $\mathrm{C}_{6}$ and the invariant 6 degree meromorphic function, one could proceed as in the previous sections using the character table of the automorphism group but, as in this case this is a cyclic group with rather low order, it is obvious that

$$
f_{5}(x, y)=x^{2}
$$

and

Lemma 30. For every Fuchsian group $\Gamma$ with signature $[2,3,3,6]$, there exists a unique non-equivalent surface kernel epimorphism $\varphi: \Gamma \rightarrow \mathrm{C}_{6}$ given by

$$
\varphi(x)=a, \quad \varphi(y)=a^{2} \text { and } \varphi(\mathrm{z})=\mathrm{a}^{2} .
$$

On the other hand, now using (6.1) we can get a representative for each of the 4 orbits of fixed points and their images under $f_{5}$ on the Riemann sphere, which are

$$
\begin{aligned}
P_{1}:=\left(0, t^{1 / 3}\right) & \longmapsto Q_{1}=0 \\
P_{2}:=(1,0) & \longmapsto Q_{2}=1 \\
P_{3}:=(\sqrt{t}, 0) & \longmapsto Q_{3}=t \\
P_{4}:=\infty & \longmapsto Q_{4}=\infty,
\end{aligned}
$$

where $P_{1}$ is one of the 3 fixed points of $a^{3}, P_{2}$ is one of the 4 fixed points of $a^{2}, P_{3}$ is a fixed point of $a^{3}$ in a different orbit than $P_{2}$ and, finally, $P_{4}$ is the unique fixed point of $a$. In this section the cross ratio of the critical values is $\lambda\left(Q_{1}, Q_{2}, Q_{3}, Q_{4}\right)=\frac{1}{1-t}=\lambda_{5}$ which implies that $t=\frac{\lambda_{5}-1}{\lambda_{5}}$ and if $\lambda\left(Q_{1}, Q_{3}, Q_{2}, Q_{4}\right)=\frac{t^{\prime}}{t^{\prime}-1}=\lambda_{5}$ then $t^{\prime}=\frac{\lambda_{5}}{\lambda_{5}-1}=\frac{1}{t}$. Therefore if we consider the order 2 Möbius transformation permuting the two critical values with ramification order equals 3 , then the identity makes commutative the group diagram in Lemma 1 corresponding to this Möbius transformation and these normal coverings, so it lifts to an isomorphism between $S_{t}$ and $S_{1 / t}$. Moreover, an isomorphism between $S_{1 / t}$ and $S_{t}$ belonging to $\mathcal{F}_{5}$ is, obviously, given by

$$
\epsilon(x, y)=\left(e x, e^{4 / 3} y\right) .
$$

On the other hand, we have

Lemma 31. The possible inclusions of a Fuchsian group $\Gamma_{i}$ of signature $[2,3,3,6]$ inside a triangle group $\Delta_{i}$ are the following:
i) $\Gamma_{1}=[2,3,3,6]_{1} \leq[3,3,6]=\Delta_{1} \unlhd[2,3,12]$ with indexes 4 and 8 , resp.
ii) $\Gamma_{2}=[2,3,3,6]_{2} \leq[2,3,30]=\Delta_{2}$ with index 5.
iii) $\Gamma_{3}=[2,3,3,6]_{3} \leq[2,5,6]=\Delta_{3}$ with index 5.
iv) $\Gamma_{4}=[2,3,3,6]_{4} \leq[2,6,9]=\Delta_{4}$ with index 3.
v) $\Gamma_{5}=[2,3,3,6]_{5} \unlhd[3,4,12]=\Delta_{5}$ with index 2. 
Proof. Again, with a simple area argument and proceeding as in Lemma 13, and using the fact that one of the periods of any Fuchsian group that contains a quadrangle group with signature $[2,3,3,6]$ must be a multiple of 6 , as $\mu([2,3,3,6])=2 / 3$ and, for every $n$ and $l \geq 4$, we have $\mu([n, l, 6 k]) \geq$ $\mu([4,4,6])=1 / 3=\mu([2,3,3,6]) / 2$. We conclude that there is no triangle group $[n, l, 6 k]$ containing a quadrangle group with signature $[2,3,3,6]$, for $n \geq l \geq 4$ and for any $k$, because Theorem 1 in [14] states that such an inclusion can not happen either for the unique possibility which was $[4,4,6]$, with index $N=2$, as one would obtain more than one six period for the included subgroup.

Analogously, $\mu([3, l, 6 k])>1 / 3=\mu([2,3,3,6]) / 2=\mu([2,3,3,6]) / N$ for any $l \geq 3$ if $k \geq 3$; and for $k=1$ and 2 the only possibilities are $[4,4,6]$ with $N=2$ which is not possible because of Theorem 1 in [14] for the same reason as for $[4,4,6]$, but $[3,3,6]$ with index $N=4$ and $[3,4,12]$ with index $N=2$ could happen.

On the other hand, we have to discuss the possible cases for $n=2$. Let us observe that $\mu([2, l, 6 k])>1 / 3=\mu([2,3,3,6]) / 2=\mu([2,3,3,6]) / N$ implies that $l \cdot(k-1)>6 k$ or, in other words, $l>\frac{6 k}{k-1}$ for $k>1$, so $l>8$ for $k \geq 4$. Hence, let us now study the possibilities for $k=1,2$ and 3:

- $\mu([2, l, 6])=\frac{l-3}{3 l}=2 /(3 N)$ or, equivalently, $N \cdot(l-3)=2 l$. Therefore, $l-3$ divides $2 l$ which implies that $l-3$ divides $6=2 \cdot(l-3)-2 l$ or, in other words, the possibilities for $l$ are 4,5,6 and 9. Using Theorem 1 in [14] we discard easily $[2,6,6]$, it is impossible to obtain a permutation representation in $S_{4}$ as the action of the generators of the triangle group on a set of left-coset representatives of the quotient $[2,6,6] /[2,3,3,6]$.

- $\mu([2, l, 12])=\frac{5 l-12}{12 l}=\frac{2}{3 N}$, hence $N \cdot(5 l-12)=8 l$ which implies that $5 l-12$ divides $8 l$ and therefore, $5 l-12$ divides $8 \times 12=96$. Then, if we check the possibilities for $l$ we only obtain the integer solutions $l=4$ and 8 .

- Finally, $\mu([2, l, 18])=\frac{16 l-9}{9 l}=\frac{2}{3 N}$ or, equivalently, $N \cdot(16 l-9)=6 l$ which implies that $16 l-9$ divides $6 l$, so $16 l-9$ divides $27=3 \times 9$, but this gives no integer solutions for $l$.

It still remains to study the possible values of $k$ for $l=3,4,5,6,7$ and 8 , and reasoning in an analogous way as above, the new candidates obtained are $[2,3,12],[2,3,18],[2,3,30],[2,4,12],[2,4,36]$ and $[2,7,42]$ for which the corresponding indexes are $N=8,6,5,2,3$ and 2 . According to Theorem 1 in [14], a subgroup contained in $[2,3,18]$ with index $N=6$ would have more than 3 periods; $[2,4,36]$ and $[2,7,42]$ can not contain a Fuchsian group with signature $[2,3,3,6]$ and with indexes 3 and 2 , respectively, because it would 
give us generators for the included subgroups with orders, at least, 12 and 21, respectively. The permutation representations that prove that, in fact, the rest of possible inclusion really happens are

$$
\begin{aligned}
& \theta_{1}:[2,3,12] \rightarrow S_{8} \\
& x_{1} \rightarrow(12)(37)(45)(68) \\
& y_{1} \rightarrow(2)(5)(173)(486) \\
& z_{1} \rightarrow(123456)(78) \\
& \theta_{1^{\prime}}:[3,3,6] \rightarrow S_{4} \\
& x_{1}^{\prime} \rightarrow(234)(1) \\
& y_{1}^{\prime} \rightarrow(142)(3) \\
& z_{1}^{\prime} \rightarrow(123)(4) \\
& \theta_{2}:[2,3,30] \rightarrow S_{5} \\
& x_{2} \rightarrow(1)(23)(45) \\
& \theta_{3}:[2,5,6] \rightarrow S_{5} \\
& y_{2} \rightarrow(3)(5)(142) \\
& x_{3} \rightarrow(1)(23)(45) \\
& z_{2} \rightarrow(12345) \\
& y_{3} \rightarrow(13542) \\
& z_{3} \rightarrow(12)(34)(5) \\
& \theta_{4}:[2,6,9] \rightarrow S_{3} \\
& x_{4} \rightarrow(1)(23) \\
& \theta_{5}:[3,4,12] \rightarrow S_{2} \\
& y_{4} \rightarrow(12)(3) \\
& x_{5} \rightarrow(1)(2) \\
& z_{4} \rightarrow(123) \\
& y_{5} \rightarrow(12) \\
& z_{5} \rightarrow(12),
\end{aligned}
$$

which concludes the proof.

Summarizing up,

Proposition 32. $S_{t}$ and $S_{t^{\prime}}$ belonging to $\mathcal{F}_{5}$ are isomorphic if and only if $p_{5}\left(t^{\prime}\right)=p_{5}(t)$, where $p_{5}: \mathbb{C P}^{1} \rightarrow \mathbb{C P}^{1}$ is given by $p_{5}(z)=z+\frac{1}{z}$.

Remark 33. $p_{5}: \mathbb{C P}^{1} \rightarrow \mathbb{C P}^{1}$ is a Belyi function with critical values \pm 2 . The algebraic curves corresponding to the set of preimages of the critical values are:

i) $p^{-1}(2)=\{1\}$ which corresponds to a singular curve as it is the case for $\{0, \infty\}=p^{-1}(\infty)$.

ii) $p^{-1}(-2)=\{-1\}$ which corresponds to the curve given by the following algebraic equation:

$$
y^{3}=x^{4}-1
$$

Its automorphism group is isomorphic to $\mathrm{C}_{12}$, generated by

$$
a^{\prime}(x, y)=\left(\sqrt{-1} x, \zeta_{3} y\right) \text { with } \zeta_{3}=\exp (2 \pi \sqrt{-1} / 3)
$$

In terms of Fuchsian groups, its surface group has to be contained in the unique normal inclusion of a Fuchsian quadrangle group with signature $[2,3,3,6]$ in a triangle group, which is $[3,4,12]$ with index 2 .

Moreover, 
Theorem 34. Let $Q_{5}$ be the order 2 group of Möbius transformations generated by $z \mapsto \frac{1}{z}$, and let $\left[S_{t}\right]$ be the isomorphism class of any genus 3 compact Riemann surface belonging to the family $\mathcal{F}_{5}$. Then the mapping

$$
\Phi: t \mapsto\left[S_{t}\right]
$$

is an holomorphic injection between the analytic space $(\mathbb{C}-\{0,1\}) / Q_{5}$ and the moduli space $\mathcal{M}_{3}$ of compact Riemann surfaces of genus 3. Its image is the subvariety of $\mathcal{M}_{3}$ consisting of those surfaces admitting an automorphism group isomorphic to $\mathrm{C}_{6}$.

Proof. As in the previous sections, it follows from [11] and Proposition 32.

Again, in order to obtain the parameter $t$ corresponding to the complete list of algebraic curves belonging to the family $\mathcal{F}_{5}$ for which their surface groups lie in the quadrangle groups $\Gamma_{i}$ inside the triangle groups listed in Lemma 31 or, equivalently, the ones which admit a uniform Belyi function that factorizes through the quotient by the automorphism group, we use the same procedure and notation as in the previous sections. In this case, we obtain the values of $t$ imposing that

$$
\frac{1}{1-t}=\lambda(0,1, t, \infty)=\lambda\left(Q_{1}, Q_{2}, Q_{3}, Q_{4}\right)=\lambda\left(R_{1}^{i}, R_{2}^{i}, R_{3}^{i}, R_{4}^{i}\right) ;
$$

namely, following the order of the cases of the triangle inclusions listed in Lemma 31 and with the previously fixed notation,

Proposition 35. A Riemann surface $S_{t} \in \mathcal{F}_{5}$ has the normalizer of its surface group contained in a triangle group or, in other words, $S_{t}$ has a uniform Belyi function which factorizes through the quotient by the automorphism group if and only if $t$ is obtained from the data of one of the following cases:

i) $h_{1}(z)=\frac{(z+1)^{3}(z-1 / 7)}{z^{3}(z-1)} ; \quad\left(\nu_{1}^{11}, \nu_{2}^{11} ; \nu_{1}^{21}, \nu_{2}^{21} ; \nu_{1}^{31}, \nu_{2}^{31}\right)=(3,1 ; 3,1 ; 3,1)$.

$R_{1}^{1}=\frac{-1}{5}, R_{2}^{1}=1, R_{3}^{1}=\frac{1}{7}$ and $R_{4}^{1}=-1$.

$\lambda\left(R_{1}^{1}, R_{2}^{1}, R_{3}^{1}, R_{4}^{1}\right)=2=\frac{1}{1-t}$ therefore $t=\frac{1}{2}$.

ii) $h_{2}(z)=z^{3}\left(z^{2}-2 z+32 / 5\right)$;

$\left(\nu_{1}^{12}, \nu_{2}^{12}, \nu_{3}^{12} ; \nu_{1}^{22}, \nu_{2}^{22}, \nu_{3}^{22} ; \nu_{1}^{32}\right)=(2,2,1 ; 3,1,1 ; 5)$.

$R_{1}^{2}=-\frac{6}{5}, R_{2}^{2}=\frac{5+3 \sqrt{-3}}{5}, R_{3}^{2}=\frac{5-3 \sqrt{-3}}{5}$ and $R_{4}^{3}=\infty$.

$\lambda\left(R_{1}^{2}, R_{2}^{2}, R_{3}^{2}, R_{4}^{2}\right)=\frac{9-11 \sqrt{-3}}{18}=\frac{1}{1-t}$ therefore $t=\frac{47-33 \sqrt{-3}}{74}$.

iii) $h_{3}(z)=\left(16 z^{5}-20 z^{3}+5 z\right)$;

$\left(\nu_{1}^{13}, \nu_{2}^{13}, \nu_{3}^{13} ; \nu_{1}^{23}, \nu_{2}^{23}, \nu_{3}^{23} ; \nu_{1}^{33}\right)=(2,2,1 ; 2,2,1 ; 5)$.

$R_{1}^{3}=-1, R_{2}^{3}=\cos (4 \pi / 5), \mathrm{R}_{3}^{3}=\cos (2 \pi / 5)$ and $\mathrm{R}_{4}^{3}=1$.

$\lambda\left(R_{1}^{3}, R_{2}^{3}, R_{3}^{3}, R_{4}^{3}\right)=\frac{2-\sqrt{5}}{4}=\frac{1}{1-t}$ therefore $t=9+4 \sqrt{5}$. 


$$
\begin{aligned}
& \text { iv) } h_{4}=\left(4 z^{2}-3\right) z ; \quad\left(\nu_{1}^{14}, \nu_{2}^{14} ; \nu_{1}^{24}, \nu_{2}^{24} ; \nu_{1}^{34}\right)=(2,1 ; 2,1 ; 3) . \\
& \quad R_{1}^{4}=1, R_{2}^{4}=\infty, R_{3}^{4}=\frac{1}{2} \text { and } R_{4}^{4}=-1 . \\
& \quad \lambda\left(R_{1}^{4}, R_{2}^{4}, R_{3}^{4}, R_{4}^{4}\right)=\frac{3}{4}=\frac{1}{1-t} \text { therefore } t=\frac{-1}{3} . \\
& \text { v) } h_{5}=z^{2} ; \quad\left(\nu_{1}^{15} ; \nu_{1}^{25}, \nu_{2}^{25} ; \nu_{1}^{35}\right)=(2 ; 1,1 ; 2) . \\
& \quad R_{1}^{5}=0, R_{2}^{5}=1, R_{3}^{5}=-1 \text { and } R_{4}^{5}=\infty . \\
& \lambda\left(R_{1}^{5}, R_{2}^{5}, R_{3}^{5}, R_{4}^{5}\right)=\frac{1}{2}=\frac{1}{1-t} \text { therefore } t=-1 .
\end{aligned}
$$

Similarly as in the previous sections for the non-hyperelliptic families, for every genus 3 curve $S_{t}$ belonging to the family $\mathcal{F}_{5}$, we obtain the 6 -holomorphic differential which is the Wronskian $\left(\operatorname{Wr}_{5}\left(S_{t}\right)\right)$ of $x, y$ and $z$, a basis of the vector space of holomorphic differentials. In order to make the direct computation which is the Wronskian for this basis, we use the meromorphic function $y / z$ as a local parameter at every point of the curve, unless a finite number of them, in the affine neighborhood $z=1$ and, finally, we return it to a global expression what gives us that

Theorem 36. For every compact Riemann surface $S_{t}$ belonging to the family $\mathcal{F}_{5}$, the Wronskian of the basis $\{x, y, z\}$ for the space of holomorphic differentials is

$$
\mathrm{Wr}_{5}\left(S_{t}\right)=-2 y^{4} x^{2}+3 y^{4} z^{2}+4 x^{2} y z^{3}+\left(3 y^{4} z^{2}-8 x^{2} y z^{3}\right) t+\left(4 x^{2} y z^{3}\right) t^{2} .
$$

\section{References}

[1] Bétréma, J. And Zvonkin. A.: Plane trees and Shabat polynomials. Proceedings of the 5th Conference on Formal Power Series and Algebraic Combinatorics (Florence, 1993). Discrete Math. 153 (1996), no. 1-3, 47-58.

[2] Broughton, S. A.: Classifying finite group actions on surfaces of low genus. J. Pure Appl. Algebra 69 (1990), no. 3, 233-270.

[3] Bujalance, E., Costa, A. F., Gromadzki, G. and Singerman, D.: Automorphism groups of complex doubles of Klein surfaces. Glasgow Math. J. 36 (1994), no. 3, 313-330.

[4] Bujalance, E., Cirre, F. J., Gamboa, J. M. and Gromadzki, G.: On compact Riemann surfaces with dihedral groups of automorphisms. Math. Proc. Cambridge Philos. Soc. 134 (2003), no. 3, 465-477.

[5] Farkas, H. And Kra, I.: Riemann surfaces. Graduate Texts in Mathematics 71. Springer-Verlag, New York-Berlin, 1980.

[6] González-Díez, G.: Loci of curves which are prime Galois coverings of $\mathbb{C P}^{1}$. Proc. London Math. Soc. (3) 62 (1991), no. 3, 469-489.

[7] González-Díez, G. And Harvey, W.: Moduli of Riemann surfaces with symmetry. In Discrete Groups and Geometry, 75-93. London Math. Soc. Lecture Note Ser. 173. Cambridge Univ. Press, Cambridge, 1992. 
[8] Jones, G. A.: Characters and surfaces: a survey. In The atlas of finite groups: ten years on (Birmingham, 1995), 90-118. London Math. Soc. Lecture Note Ser. 249. Cambridge Univ. Press, Cambridge, 1998.

[9] Harvey, W. H.: On branch loci in Teichmüller space. Trans. Amer. Math. Soc. 153 (1971), 387-399.

[10] Kuribayashi, A. And Komiya, K.: On the Structure of the Automorphism Group of a Compact Riemann Surface of Genus 3. Bull. Facul. Sci. and Eng. Chou University 23 (1980), 1-34.

[11] NAG, S.: The Complex Analytic Theory of Teichmüller Spaces. Wiley, New York, 1988.

[12] Rodríguez, R. E. And Gónzalez-Aguilera, V.: Fermat quartic curve, Klein's curve and the tetrahedron. Contemp. Math. 201 (1997), 43-62.

[13] Rohrlich, D. E.: Some remarks on Weierstrass points. In Number Theory Related to Fermat's Last Theorem, 71-78. Progr. Math. 26. Birkhäuser, Basel, 1982.

[14] Singerman, D.: Subgroups of Fuchsian Groups and Finite Permutation Groups. Bull. London Math. Soc. 2 (1970), 319-323.

[15] Singerman, D. And Syddhall, R.: Belyi Uniformization of Elliptic Curves. Bull. London Math. Soc. 139 (1997), 443-451.

[16] Streit, M.: Homology, Belyi Functions and Canonical Curves. Manuscripta Math. 90 (1996), 489-509.

[17] Streit, M. And Wolfart, J.: Characters and Galois invariants of regular dessins. Rev. Mat. Complut. 13 (2000), no. 1, 49-81.

[18] Wiman, A.: Über die hyperelliptischen Kurven und diejenigen vom Geschlechte $p=3$ welche eindeutige Transformationen in sich zulassen. Bihang Till Kungl. Svenska Vet. Akad. Handlingar 21 (1895), no. 1, 1-23.

[19] Zvonkin, A.: Megamaps: constructions and examples. In Discrete models: Combinatorics, Computation and Geometry (Paris, 2001), 329-339. Discrete Math. Theor. Compt. Sci. Proc. AA. Maison Inform. Math. Discret., Paris, 2001.

Recibido: 20 de enero de 2004

Yolanda Fuertes

Departamento de Matemáticas Universidad Autónoma de Madrid Madrid, 28049, España yolanda.fuertes@uam.es

Manfred Streit manfred.streit@tlc.de 